\title{
Energy transfer mechanisms in layered 2D perovskites
}

\author{
Olivia F. Williams, ${ }^{\text {a) }}$ Zhenkun Guo, ${ }^{\text {a) }}$ Jun Hu, Liang Yan, Wei You, and Andrew M. Moran ${ }^{\text {b) }}$ \\ Department of Chemistry, University of North Carolina at Chapel Hill, Chapel Hill, North Carolina 27599, USA
}

(Received 18 October 2017; accepted 16 March 2018; published online 4 April 2018)

\begin{abstract}
Two-dimensional (2D) perovskite quantum wells are generating broad scientific interest because of their potential for use in optoelectronic devices. Recently, it has been shown that layers of 2D perovskites can be grown in which the average thicknesses of the quantum wells increase from the back to the front of the film. This geometry carries implications for light harvesting applications because the bandgap of a quantum well decreases as its thickness increases. The general structural formula for the $2 \mathrm{D}$ perovskite systems under investigation in this work is (PEA) $)_{2}(\mathrm{MA})_{\mathrm{n}-1}\left[\mathrm{~Pb}_{\mathrm{n}} \mathrm{I}_{3 \mathrm{n}+1}\right](\mathrm{PEA}$ $=$ phenethyl ammonium, $\mathrm{MA}=$ methyl ammonium). Here, we examine two layered $2 \mathrm{D}$ perovskites with different distributions of quantum well thicknesses. Spectroscopic measurements and model calculations suggest that both systems funnel electronic excitations from the back to the front of the film through energy transfer mechanisms on the time scales of 100's of ps (i.e., energy transfer from thinner to thicker quantum wells). In addition, the model calculations demonstrate that the transient absorption spectra are composed of a progression of single exciton and biexciton resonances associated with the individual quantum wells. We find that exciton dissociation and/or charge transport dynamics make only minor contributions to the transient absorption spectra within the first $1 \mathrm{~ns}$ after photoexcitation. An analysis of the energy transfer kinetics indicates that the transitions occur primarily between quantum wells with values of $n$ that differ by 1 because of the spectral overlap factor that governs the energy transfer rate. Two-dimensional transient absorption spectra reveal a pattern of resonances consistent with the dominance of sequential energy transfer dynamics. Published by AIP Publishing. https://doi.org/10.1063/1.5009663
\end{abstract}

\section{INTRODUCTION}

Two-dimensional (2D) perovskites are generating broad scientific interest because of their potential for use in optoelectronic devices. ${ }^{1-7}$ These systems are generally described by the formula $A_{n}(M A)_{n-1} M_{n} Y_{3 n+1}$, where $A$ is an organic cation, $\mathrm{MA}$ is methylammonium $\left(\mathrm{CH}_{3} \mathrm{NH}_{3}+\right), \mathrm{M}$ is the metal cation, and $\mathrm{Y}$ is the halide. Lead iodide systems are among the most commonly studied 2D perovskites and will be the focus of the present work. It has been shown that fairly large organic cations (such as phenethyl ammonium) confine excitons to 2D lead iodide layers, which gives rise to binding energies as high as $380 \mathrm{meV}$ for a quantum well with one lead iodide sheet $(n=1){ }^{6}$ The exciton binding energies decrease as $n$ increases but are still much larger than $\mathrm{k}_{\mathrm{B}} \mathrm{T}$ at room temperature for $\mathrm{n}=5$ (the exciton binding energy is estimated to be $235 \mathrm{meV}$ for $\mathrm{n}=5){ }^{6}$ The understanding of these materials is most complete for quantum wells composed of 1-2 leadhalide layers. ${ }^{8-12}$ However, electronic processes in thicker 2D perovskite quantum wells are presently under investigation by a number of groups. ${ }^{6,13-15}$

It has recently been discovered that layered 2D perovskites can be engineered to funnel energy and/or charge carriers from thinner to thicker quantum wells located on opposite sides of the film. ${ }^{15}$ In this type of system, the quantum wells stack

\footnotetext{
a)O. F. Williams and Z. Guo contributed equally to this work.

b)Author to whom correspondence should be addressed: ammoran@ email.unc.edu
}

in the order of increasing thickness. ${ }^{15}$ Because the bandgap of a quantum well decreases as the thickness increases [see Fig. 1(a)], ${ }^{6}$ such layered systems are capable of directing energy transfer in a manner similar to natural light-harvesting antenna. ${ }^{16}$ Recent studies have recognized the propensity for energy transfer in layered quantum wells. ${ }^{17,18}$ However, it has also been suggested that electron and hole transfer occur in parallel with energy transfer. ${ }^{15,19}$ The dynamics in these systems have been interpreted in the framework of the morphologies depicted in Fig. 1. In the quasi-layered structure of Fig. 1(b), the average size of the quantum well increases with respect to height in the film, whereas a distribution of quantum well sizes exists within each layer [see Fig. 1(c)]. Thus, electronic excitations may transfer laterally and vertically following photo-excitation; however, the excitations ultimately concentrate in the $n=\infty$ layer which is located on the front of the film.

The structural formula for the $2 \mathrm{D}$ perovskite under investigation in this work is (PEA) $2_{2}(\mathrm{MA})_{\mathrm{n}-1}\left[\mathrm{~Pb}_{\mathrm{n}} \mathrm{I}_{3 \mathrm{n}+1}\right]$ (PEA $=$ phenethyl ammonium). The value of $\mathrm{n}$ determines the thickness and resonance frequency of the quantum well. For example, quantum wells with $\mathrm{n}=2,3$, and 4 exhibit excitonic resonances near 570, 610, and $645 \mathrm{~nm}$. In this system, quantum confinement is enforced by the 0.9 -nm thick layers of PEA that separate the lead iodide quantum wells. ${ }^{10,11}$ We investigate two types of films in the present work and introduce a notation to distinguish the systems. For the purpose of discussion, we will hereafter denote the quantum well whose concentration is optimized by chemical preparation with $N_{\max }\left(N_{\max }=2\right.$ 

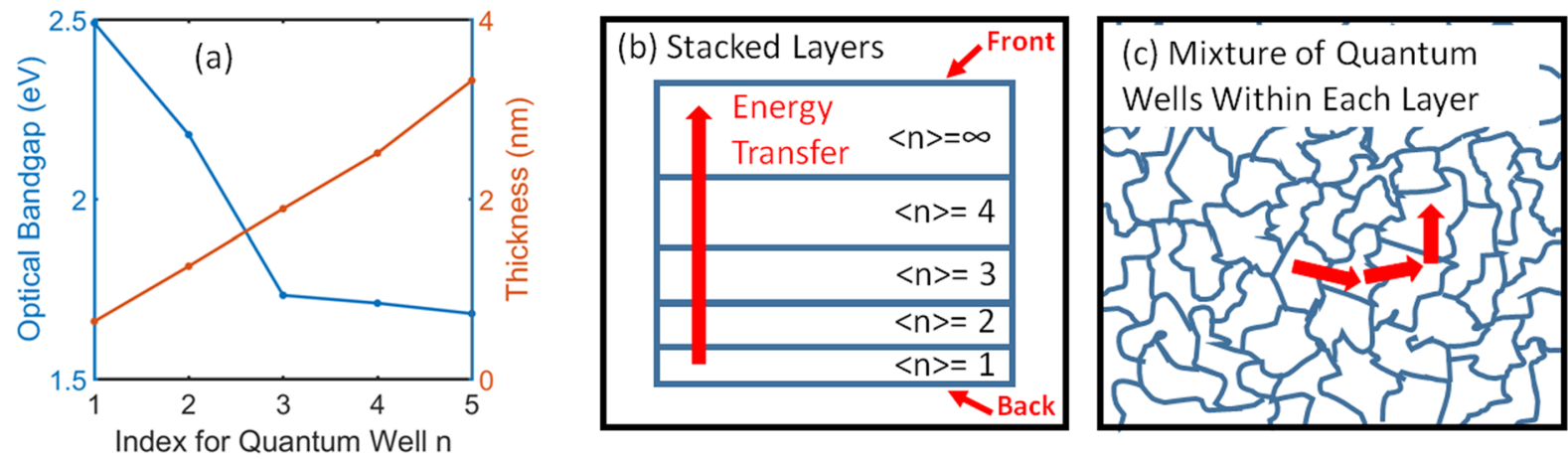

FIG. 1. (a) Optical bandgaps and thicknesses of the quantum wells plotted as a function of $\mathrm{n}$ for a system similar to that investigated in this work (i.e., a system with a butylammonium organic cation instead of PEA). ${ }^{6}$ (b) The average thicknesses of the quantum wells increase with the distance from the substrate. Energy transfer is directed from the back to the front of the film because of variation in the average bandgap. The smallest quantum wells are located at the back of the film near the substrate surface, whereas a bulk-like layer is located at the front of the film. (c) Within each layer, electronic excitations may migrate among quantum wells with different values of $n$.

or 3). The indices of the individual quantum wells are written in lower case, $n$. The reason for this distinction is that chemical preparation may be used to optimize the concentration of a particular quantum well, $n$, but others (such as $n-1$ and $\mathrm{n}+1$ ) are also produced in the process. One of the films examined in the present work is nominally $\mathrm{N}_{\max }=2$ but possesses resonances associated with several sizes of quantum wells $(\mathrm{n}=2,3,4,5$, and $\infty)$. The second film is nominally $\mathrm{N}_{\max }=3$ but similarly exhibits multiple excitonic transitions.

The dynamics of electronic excitations in these 2D perovskites are studied with steady-state and time-resolved optical measurements. The layered morphology is established by conducting steady-state emission and transient absorption experiments with light incident on both sides of the film. Rate constants associated with energy transfer between pairs of quantum wells are obtained by fitting transient absorption data with a global kinetic model in a basis of single excitons and biexciton states. Our assignment of the dynamics to an energy transfer mechanism is shown to be consistent with the line shapes of the transient absorption spectra and Förster's rate formula. ${ }^{20,21}$ Finally, two-dimensional transient absorption (2DTA) spectra are used to inspect correlations between the initial locations of electronic excitations and the locations at the delay time, $\tau$. The pattern of peaks in the 2DTA spectra also supports a sequential energy transfer mechanism between quantum wells whose indices differ by 1 .

\section{EXPERIMENTAL}

\section{A. Sample preparation and characterization}

Two-dimensional perovskite films are prepared using previously reported methods. ${ }^{13}$ Phenethyl ammonium iodide and dimethylformamide (DMF) are obtained from Sigma-Aldrich, lead iodide is purchased from Alfa Aesar, and methylammonium iodide is obtained from Solaronix. All materials are used without further purification.

FTO (Sigma-Aldrich; $13 \Omega /$ sq, approx. $4 \mathrm{~cm}^{2}$ ) substrates are cleaned via sonication for $20 \mathrm{~min}$ in a series of four solvents: Contrex AP glassware detergent in deionized water, deionized water, acetone, and isopropanol. Substrates are dried in a stream of nitrogen gas following the isopropanol wash and preheated at $100{ }^{\circ} \mathrm{C}$ for 20 min immediately before film deposition. For SEM and Powder X-Ray Diffraction (PXRD) experiments, thinner FTO substrates (MTI; 7-10 $\Omega /$ sq, 1 sq. in.) were prepared in the same way before thin film deposition.

These 2D perovskites are described by the general formula $(\mathrm{PEA})_{2}(\mathrm{MA})_{\mathrm{n}-1}\left[\mathrm{~Pb}_{\mathrm{n}} \mathrm{I}_{3 \mathrm{n}+1}\right]$, where $\mathrm{n}$ is the number of lead iodide layers between the organic cation spacers. Perovskites with two lead iodide layers (a film that is nominally $\mathrm{N}_{\max }=2$ ) are produced using a 2:1:2 molar ratio of PEAI, $\mathrm{MAI}$, and $\mathrm{PbI}_{2}$, respectively. The (PEA) $)_{2}(\mathrm{MA})\left[\mathrm{Pb}_{2} \mathrm{I}_{7}\right]$ precursor is prepared by dissolving PEAI (0.288 g), MAI (0.092 g), and $\mathrm{PbI}_{2}(0.533 \mathrm{~g})$ in DMF $(2 \mathrm{ml})$. Perovskite samples that are nominally $\mathrm{N}_{\max }=3$ are produced using a 2:2:3 molar ratio of $\mathrm{PEAI}, \mathrm{MAI}$, and $\mathrm{PbI}_{2}$, respectively. A precursor solution for $(\mathrm{PEA})_{2}(\mathrm{MA})_{2}\left[\mathrm{~Pb}_{3} \mathrm{I}_{10}\right]$ is prepared by dissolving PEAI (0.288 g), MAI (0.184 g), and $\mathrm{PbI}_{2}(0.800 \mathrm{~g})$ in DMF (2 ml). Films are prepared by spin coating $100 \mu \mathrm{l}$ of respective precursor solution at $3000 \mathrm{rpm}$ for $20 \mathrm{~s}$ (1500 rpm/s acceleration) onto the clean, preheated substrate.

Film thicknesses and grain sizes are determined using SEM (see the supplementary material). The $\mathrm{N}_{\max }=2$ and $\mathrm{N}_{\text {max }}=3$ systems have average thicknesses of 550 and $685 \mathrm{~nm}$, respectively. SEM measurements are conducted with a Hitachi S-4700 cold cathode field emission scanning electron microscope. Accelerating voltages $(\mathrm{kV})$, working distances $(\mathrm{mm})$, and magnifications, respectively, are listed in the bottom left of individual images. Powder X-Ray Diffraction (PXRD) measurements are conducted on intact, oriented layered perovskite films using a Rigaku Smartlab X-ray diffractometer in the Bragg-Brentano geometry, equipped with a $\mathrm{Cu}$ source and $\mathrm{K} \beta$ filter. All XRD measurements are recorded at room temperature in ambient conditions.

\section{B. Transient absorption spectroscopy}

All experiments are conducted with a 45-fs, 4-mJ Coherent Libra with a $1-\mathrm{kHz}$ repetition rate. Approximately $1.5 \mathrm{~mJ}$ of the 800-nm fundamental is focused into a 4-m long tube filled with argon gas to generate a visible continuum. The continuum pulse is then passed through an all-reflective 4F setup, which is based on a $1200-\mathrm{g} / \mathrm{mm}$ grating and $20-\mathrm{cm}$ focal length 
mirror. The desired portion of the spectrum is filtered with a motorized slit at the Fourier plane. The spectrally filtered pulses have 5-nm widths and 250-fs durations. The pulse energies are controlled with a neutral density filter on a motorized rotation stage.

Continuum probe pulses are generated in a sapphire window and relayed to the sample with reflective optics. The spot size of the probe is adjusted to match the $200-\mu \mathrm{m}$ spot size of the pump. The pump fluence is $2.9 \times 10^{13}$ photons $/ \mathrm{cm}^{2}$ $\left(10 \mu \mathrm{J} / \mathrm{cm}^{2}\right)$ unless indicated otherwise below. Signal detection is accomplished with a CMOS array detector that is synchronized to the $1-\mathrm{kHz}$ repetition rate of the laser system. In transient absorption experiments, the signals are averaged over 30 scans of the delay line and a total of 6000 differences are collected at each delay point.

\section{Two-dimensional transient absorption spectroscopy}

2DTA spectra can be generated using a photon-echo pulse sequence in which the full bandwidth of the pump pulse is compressed. ${ }^{22-26}$ This Fourier transform approach carries the advantage of linewidth-limited time and frequency resolution. Studies of vibrational coherence and other subpicosecond dynamics require use of broadband laser pulses. Fourier transform 2D spectra of samples with small optical densities have been measured with few-cycle continuum laser pulses. ${ }^{27,28}$ However, the Fourier transform approach is susceptible to artifacts when highly absorbing samples are investigated. ${ }^{29,30}$ For this reason, color tunable pump pulses should be considered when slower processes are under investigation. Such frequency-domain 2D methods have been successfully employed in previous $2 \mathrm{D}$ electronic and vibrational spectroscopies. ${ }^{31-34}$

In this work, the excitation axes of 2DTA spectra are generated with tunable narrowband (5-nm FWHM) pump pulses. The narrowband pump pulses are nearly transform-limited because the dispersion accumulated in transmissive optics has a negligible effect on their durations. In addition, the translation stage that controls the delay time is programmed to compensate for the dispersion in time-zero associated with the pump pulses. Thus, as in conventional TA experiments, chirp in the continuum probe pulse is the limiting factor that decides time-resolution. Dynamics that occur over hundreds of picoseconds are of interest in this study. A numerical chirp correction for the probe is not applied because the probe pulse duration (500 fs) is short compared to the relaxation dynamics under investigation.

2DTA spectra are acquired by scanning the wavelengths of pump pulses with 5-nm spectral widths from 500 to $700 \mathrm{~nm}$ in 3-nm steps. This is accomplished by translating a slit in the 4F setup described above. The schematic in Fig. 2 captures the general idea; the colors of narrowband pump pulses are scanned such that each pulse arrives at the sample at the same time via delay compensation provided by the translation stage. We have confirmed that reducing the bandwidth of the pump pulse does not decrease the spectroscopic linewidths observed by 2DTA. Thus, the measured spectral linewidths primarily represent line broadening (not the laser bandwidth). Signals are averaged for 30 scans of both the pump wavelength and delay time.

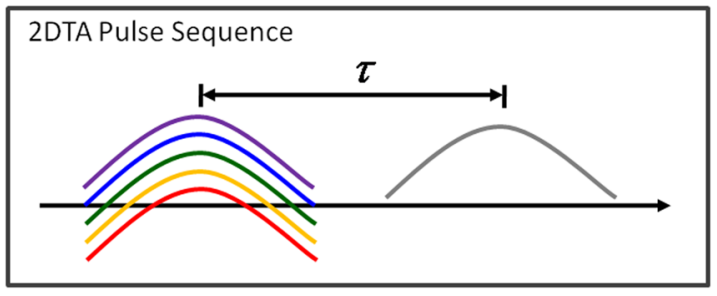

FIG. 2. Two-dimensional transient absorption (2DTA) spectra are generated by tuning 250-fs laser pulses from 500 to $700 \mathrm{~nm}$ and probing with a white-light continuum. Differential absorption is measured at each pump wavelength. The delay between the pump and probe pulses is $\tau$.

2DTA experiments are conducted using the same setup employed in the traditional transient absorption experiments described in Sec. II B. The continuum probe pulse is generated in a sapphire window and relayed to the sample with reflective optics. The pump fluence is $2.9 \times 10^{13}$ photons $/ \mathrm{cm}^{2}$ $\left(10 \mu \mathrm{J} / \mathrm{cm}^{2}\right)$. The pump and probe pulses are, respectively, chopped at 500 and $250 \mathrm{~Hz}$ in order to cycle over four conditions: pump on/probe off $\left(S_{1}\right)$, pump on/probe on $\left(S_{2}\right)$, pump off/probe off $\left(\mathrm{S}_{3}\right)$, and pump off/probe on $\left(\mathrm{S}_{4}\right)$. The transient absorption response is then computed using $\Delta \mathrm{A}=\log \left(\mathrm{S}_{2}-\mathrm{S}_{1}\right)$ $-\log \left(\mathrm{S}_{3}-\mathrm{S}_{4}\right)$. Use of two choppers increases the data acquisition time but fully eliminates pump scatter which produces artifacts on the diagonal of the 2DTA spectra. Signal detection is accomplished with a CMOS array detector that is synchronized to the $1-\mathrm{kHz}$ repetition rate of the laser system. Each point in the 2DTA spectra represents a total of 6000 differences.

\section{RESULTS AND DISCUSSION}

\section{A. Steady-state absorption and emission spectra}

The linear absorbance spectra shown in Fig. 3(a) exhibit prominent resonances at 515,570 , and $610 \mathrm{~nm}$. These peaks correspond to the $\mathrm{n}=1,2$, and 3 quantum wells. In both cases, these narrow peaks are superimposed on a broad feature associated with scattering and absorption above the bandgap. Fluorescence emission is collected from both sides of the film with excitation at $450 \mathrm{~nm}$. In all cases, emission is collected from the side of the film on which light is incident (i.e., a backscattering geometry). Following the convention of Liu et al., experimental geometries in which light is incident on the glass substrate (smallest quantum wells) and the opposite side of film (largest quantum wells) are denoted as back-side and front-side excitation, respectively (see Fig. 1). ${ }^{15}$ For both systems, emission from the back of the film is blue-shifted with respect to that collected from the front. Narrow peaks are observed at wavelengths shorter than $650 \mathrm{~nm}$ with back-side excitation and detection, whereas broad emission centered in the 700-750 nm range dominates for the front of the film.

Differences in the emission spectra measured for the back and front of the sample demonstrate that the various quantum wells $(\mathrm{n}=1,2,3, \ldots)$ are not uniformly distributed throughout the film. Rather, the measurements indicate that the average sizes of the quantum wells increase with distance from the substrate as suggested in Fig. 1(b). As in any absorptive sample, the line shapes of the emission spectra are affected by re-absorption of light as it propagates out of the 

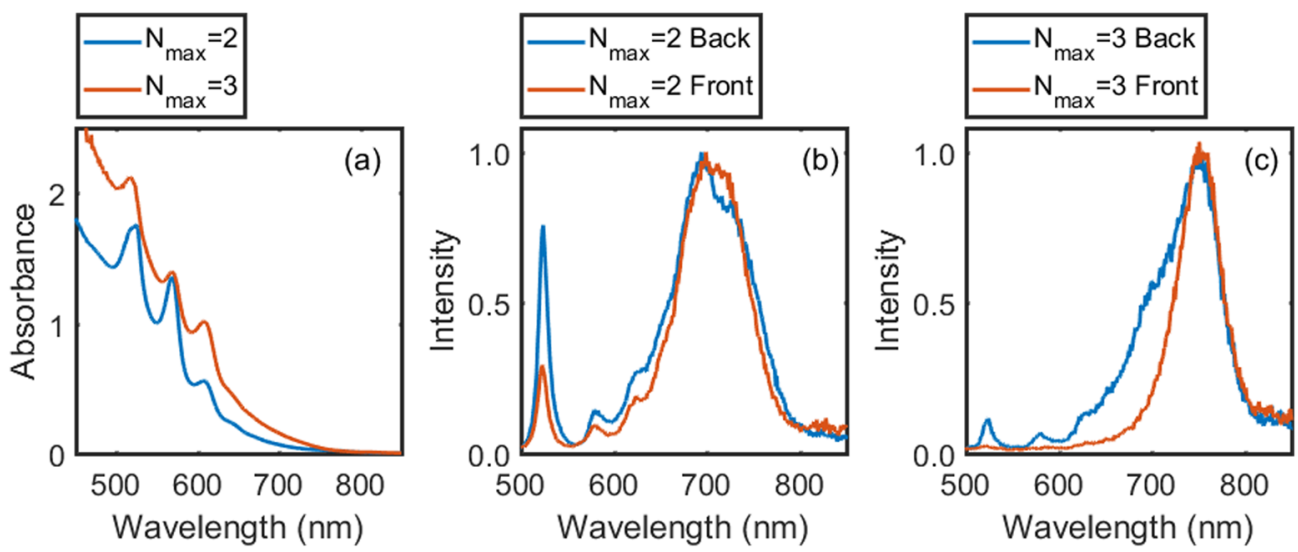

FIG. 3. (a) Linear absorbance spectra for nominal $\mathrm{N}_{\max }=2$ and $\mathrm{N}_{\max }=3$ films. (b) Steady-state emission spectra measured for the back and front of $\mathrm{N}_{\max }=2$ film. (c) Steady-state emission spectra measured for the back and front of $\mathrm{N}_{\max }=3$ film. Observation of front-side emission intensity at longer wavelengths in (b) and (c) is consistent with a layered geometry [see Fig. 1(c)].

medium. However, the effect of re-absorption on the emission spectra would be identical for the back and front of the film if the quantum wells were homogeneously distributed. Moreover, we have determined that the differences between spectra acquired for the back and front of the film are not artifacts associated with heterogeneity across the face of the film. First, these measurements average over a large number of individual grains because the spot size of the lamp on the sample $(\mathrm{FWHM}=1 \mathrm{~cm})$ is large compared to that of an individual grain ( $1 \mu \mathrm{m}$; see the supplementary material). Second, these results have been reproduced for multiple films fabricated using the methods described above (by our group and in the previous literature).${ }^{15}$ Third, in order to corroborate the results presented in Fig. 3, transient absorption signals collected for the back and front of the film, which are consistent with Fig. 1(b), are presented in the supplementary material.

The relation between the average bandgap and distance from the substrate holds implications for energy and charge transport. ${ }^{15}$ This geometry will promote directional energy transfer from the back (smallest $\mathrm{n}$ ) to the front (largest $\mathrm{n}$ ) of the film. In addition, Liu et al., who arrived at a similar conclusion for the layered geometry, have shown that hole transfer occurs in the opposite direction (front to back). ${ }^{15}$ Of course, it should be acknowledged that the schematic in Fig. 1 is an idealized version of the system. The film exhibits disorder on the micrometer length scale, which is small compared to the spot sizes of light sources employed in this work.

\section{B. Decomposition of transient absorption signal components}

Transient absorption spectra will be interpreted in this work using a response function formalism in which the signal is decomposed into three parts: (i) ground state bleach (GSB), (ii) excited state emission (ESE), and (iii) excited state absorption (ESA). ${ }^{22}$ We employ the basis set presented in Fig. 4 in which three levels (two electronic resonances) are associated with each quantum well. The energy gap between the ground state and single exciton state is denoted as $\omega_{e g, n}$, whereas the energy gap between the single exciton and biexciton states is given by $\omega_{f e, n}$. In this notation, the value of $n$ represents the specific quantum well $(\mathrm{n}=1,2,3, \ldots)$.
Notably, the excited state resonance in the $\mathrm{n}=\infty$ layer, $\omega_{f e, \infty}$, is most likely associated with free-carrier absorption within the conduction band because of its broad linewidth and the small binding energy in the bulk perovskite (see Sec. III D).

The transient absorption spectra shown in Fig. 4 exhibit progressions of resonances with alternating signs. Similar spectra have been observed in perovskite nanoparticles, ${ }^{35}$ metal dichalcogenides, ${ }^{36-38}$ and molecular aggregates. ${ }^{39,40}$ For example, transient absorption spectra for perovskite nanoparticles were modeled under the assumption of a Stark effect associated with the formation of charge transfer excitons in recent work. ${ }^{35}$ In this interpretation, the Wannier exciton produced by absorption of the pump pulse rapidly transitions into a charge transfer exciton in which the hole and electron are bound but separated by an interface between distinct phases. The electric field associated with this dipole may then perturb the resonance frequency associated with photo-excitation of a second exciton, thereby producing peaks with positive signs $(\Delta \mathrm{A})$ that are either red-shifted or blue-shifted from those of the single excitons. Notably, this is not the only mechanism by which a biexciton resonance may be shifted from that of a single exciton. Progressions of single exciton and biexciton resonances are known to arise from the quasi-fermionic nature of the excitons in ordered molecular aggregates (i.e., an effect

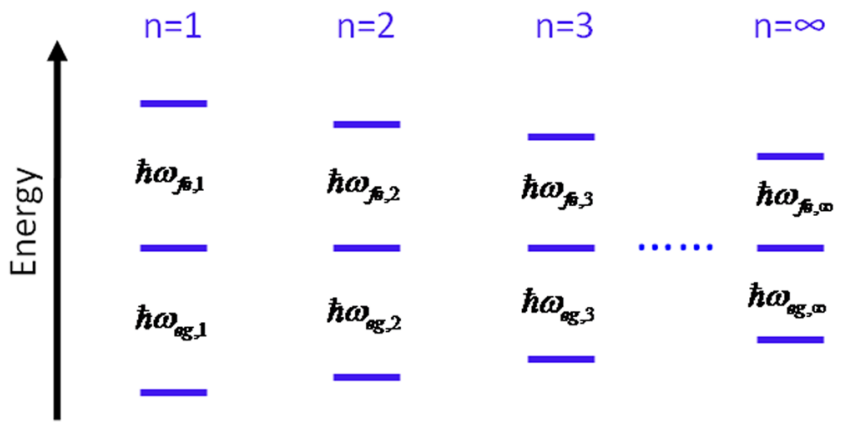

FIG. 4. The transient absorption spectrum can be decomposed into single exciton $\left(\omega_{e g, n}\right)$ and biexciton $\left(\omega_{f e, n}\right)$ resonances associated with each quantum well. This schematic does not represent the relative energies of the valence or conduction bands. 
of confinement). ${ }^{39,40}$ That is, the blue-shift of the biexciton resonance with respect to that of a single exciton is associated with the constraint that only one exciton can reside on an individual site. For this reason, the second exciton (biexciton) is confined to a smaller region of space than the first, which gives rise to a blue-shift in its resonance frequency.

The transient absorption spectra in Fig. 5 are decomposed into the GSB, ESE, and ESA components using the model outlined in Appendix A. The GSB and ESE signal components have identical line shapes because energy transfer dominates the optical response. Although the $n=2$ quantum well is targeted by photo-excitation at $570 \mathrm{~nm}$, each quantum well exhibits GSB and ESE responses at sub-picosecond delay times because a portion of the pump pulse is transmitted through the entire film. Each of the biexciton resonances in the ESA is blue-shifted from those of the single excitons by approximately $80 \mathrm{meV}$. As mentioned above, these

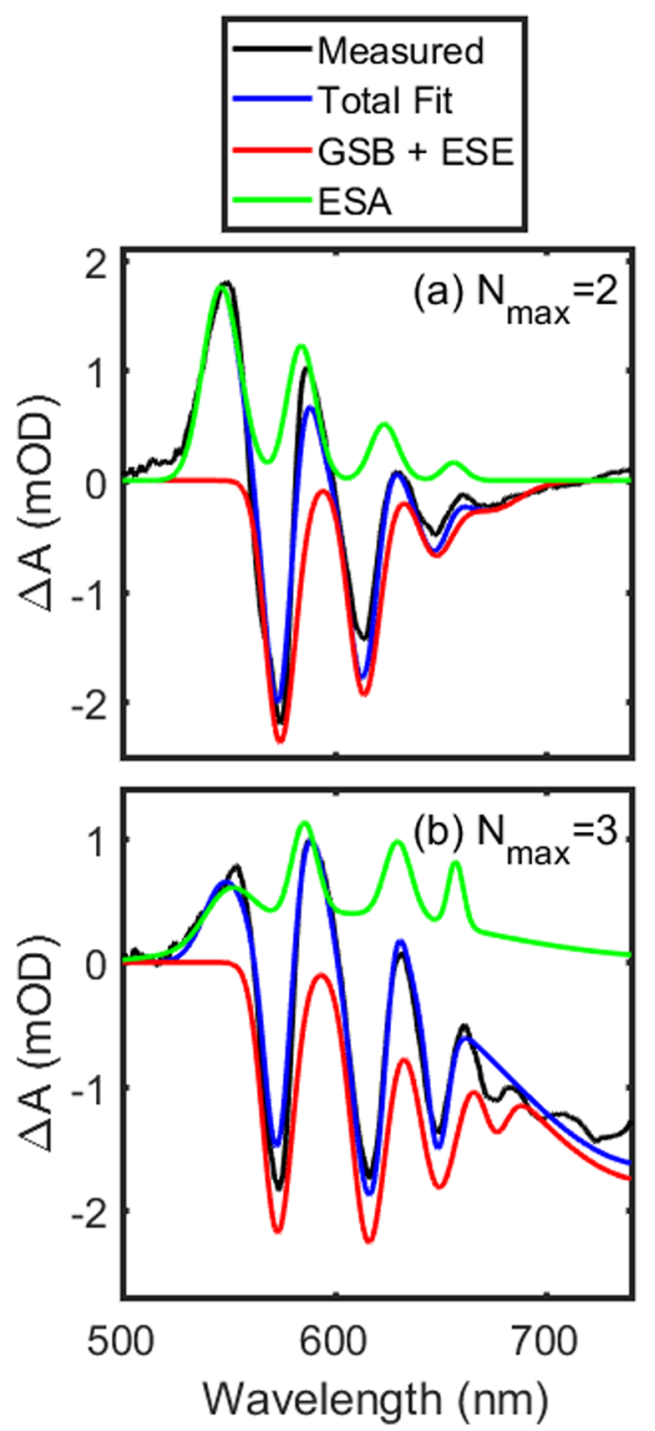

FIG. 5. Transient absorption spectra of the (a) $\mathrm{N}_{\max }=2$ and (b) $\mathrm{N}_{\max }=3$ films are decomposed into GSB, ESE, and ESA signal components using the model outlined in Appendix A. The GSB and ESE signal components have identical line shapes. The pump wavelength is $570 \mathrm{~nm}$ and the delay time is 1 ps. The parameters given in Tables I and II are constrained by a global fit to spectra at all pump-probe delay times. The laser fluence is $10 \mu \mathrm{J} / \mathrm{cm}^{2}$. blue-shifts may be associated with charge transfer excitons located at the interfaces between distinct phases. ${ }^{35}$ In an alternate interpretation, the blue-shift of the biexciton resonances represents a confinement effect similar to that found in ordered molecular aggregates. ${ }^{39,40}$ Notably, such an effect is independent of the delay time and would explain why the resonance frequencies do not vary over a $1.5 \mathrm{~ns}$ delay time. In addition, the narrow linewidths of the biexciton resonances are inconsistent with a Stark effect induced by charge transfer excitons located at interfaces. That is, a distribution in the electric field strength associated with the distance from the source of the electric field (e.g., a charge transfer exciton) would be reflected in the inhomogeneous linewidths of the biexciton resonances; however, the linewidths of the single excitons and biexcitons are quite similar, which is more consistent with a blue-shift induced by quantum confinement.

\section{Intensity-dependent signatures of many-body relaxation processes}

Classes of relaxation processes are distinguished by the order of the population, $p$, upon which they depend. ${ }^{41} \mathrm{~A}$ general differential equation for population dynamics can be written as

$$
\frac{d p}{d \tau}=-k_{1} p-k_{2} p^{2}-k_{3} p^{3}-\cdots
$$

where $k_{1}, k_{2}$, and $k_{3}$ describe relaxation rates for singlebody, two-body, and three-body processes, respectively. The linear term is primarily associated with non-radiative trapassisted recombination in perovskites. ${ }^{41,42}$ The quadratic term represents density-dependent radiative charge recombination, which occurs when an electron and hole enter a "capture radius." 43 This mechanism is prominent in $3 \mathrm{D}$ perovskites where the photoluminescence quantum yield is known to increase with the intensity of incident light. ${ }^{44-46}$ The cubic term is associated with Auger recombination of charge carriers. ${ }^{47,48}$ Auger recombination describes a process in which recombination of an electron and hole is accompanied by energy and momentum transfer to a third particle.

Intensity-dependencies of the dynamics in the $\mathrm{N}_{\max }=2$ and $\mathrm{N}_{\max }=3$ systems are illustrated in Fig. 6 . In all cases, the dynamics are initiated by absorption of 570-nm laser pulses (resonant with the $n=2$ quantum well). Variation of the laser fluence weakly impacts the decay profiles with 570-nm detection in both systems; however, the relaxation dynamics are fairly sensitive to the laser intensity at a 610-nm detection wavelength ( $\mathrm{n}=3$ quantum well). With signal detection at $645 \mathrm{~nm}(\mathrm{n}=4$ quantum well), significant intensity-dependence is found for the $\mathrm{N}_{\max }=3$ system but not for $\mathrm{N}_{\max }=2$; however, firm conclusions cannot be drawn regarding the $n=4$ quantum well in the $\mathrm{N}_{\max }=2$ film because limited signal-to-noise ratio obscures the observation (this is caused by the small concentration of $n=4$ quantum well in the film). The observed intensity dependencies of the decay profiles, particularly in the larger quantum wells, indicate that a many-body, density-dependent process contributes at larger fluences. 


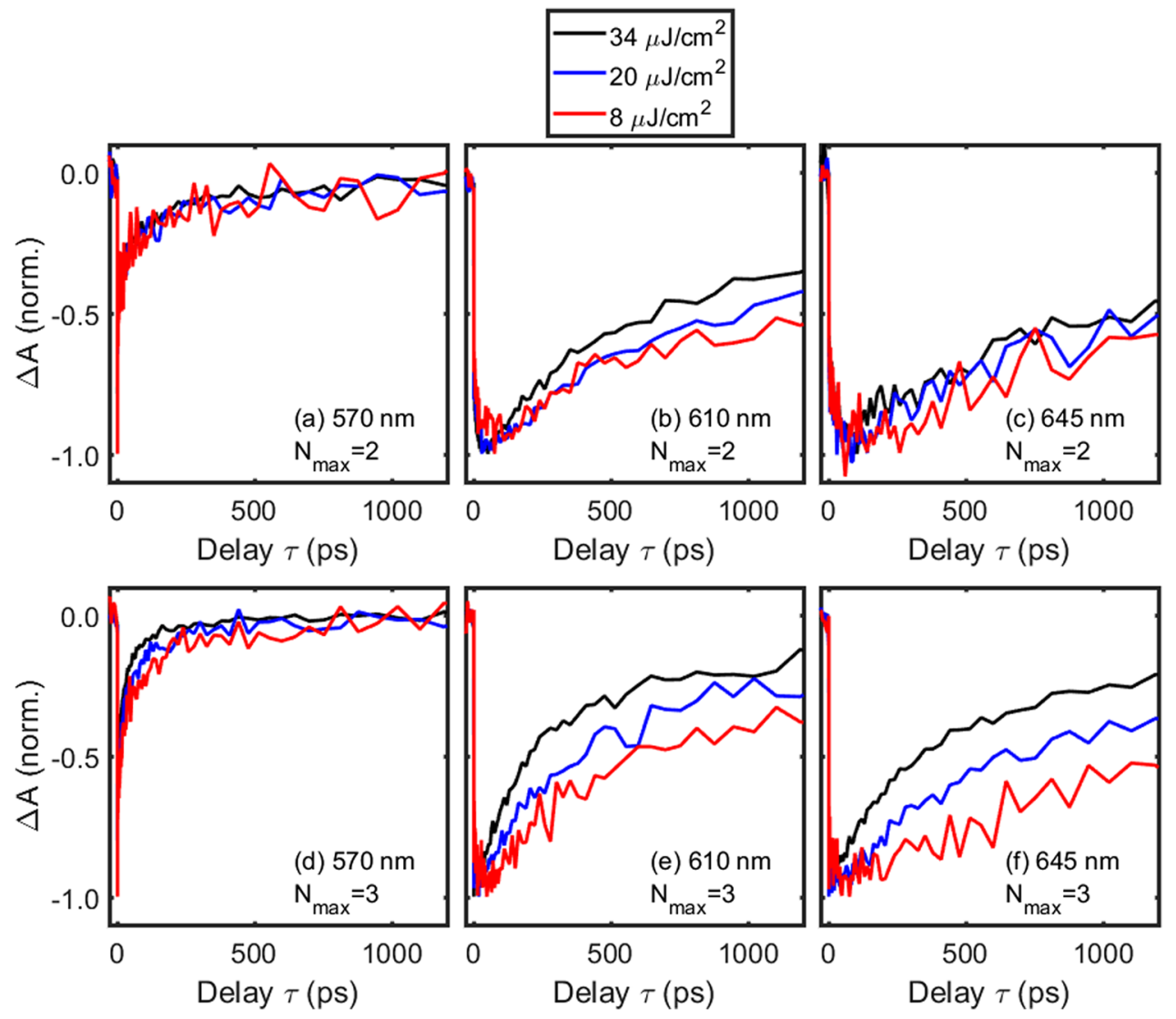

FIG. 6. Transient absorption signals measured for nominal $\mathrm{N}_{\max }=2$ (top) and $\mathrm{N}_{\max }=3$ (bottom) systems with photo-excitation at $570 \mathrm{~nm}$. Detection wavelengths are [(a) and (d)] $570 \mathrm{~nm}$; [(b) and (e)] $610 \mathrm{~nm} ;[$ (c) and (f)] $645 \mathrm{~nm}$. These wavelengths correspond to the $\mathrm{n}=2,3$, and 4 quantum wells.

The intensity-dependent dynamics found in the $\mathrm{n}=3$ and $\mathrm{n}=4$ quantum wells suggest that many-body decay processes are at work. Exciton dissociation may be promoted by the smaller binding energies found in the thicker quantum wells and/or the non-equilibrium conditions associated with the nonradiative transitions. In Fig. 7, the same types of measurements are repeated with a 610-nm excitation wavelength in order to eliminate the $\mathrm{n}=2$ to $\mathrm{n}=3$ energy transfer process from consideration. In these experiments, the $n=2$ quantum well does not exhibit GSB and ESE responses because it is transparent at $610 \mathrm{~nm}$. Again, the $\mathrm{n}=3$ and $\mathrm{n}=4$ quantum wells both exhibit intensity-dependent relaxation dynamics, although the intensity dependence for the $\mathrm{n}=3$ quantum well is notably less pronounced than that displayed in Fig. 6. Nonetheless, the observation of intensity dependence for the $\mathrm{n}=3$ quantum well in Fig. 7 suggests that spontaneous exciton dissociation takes place at larger fluences despite binding energies in excess of $235 \mathrm{meV}$ for $\mathrm{n}<6 .^{6}$ Moreover, these measurements suggest that excess thermal energy is not required to induce exciton dissociation as the pump laser is tuned into the resonance frequency of the $n=3$ quantum well (as opposed to excitation at higher energy).

Variation in the laser intensity suggests that exciton dissociation in the $n=3$ and $n=4$ quantum wells may be partly relaxation-induced (i.e., not thermally activated). In both systems, relaxation dynamics in the $\mathrm{n}=2$ quantum well, which is directly photoexcited, are relatively insensitive to the laser fluence (see Fig. 6). With photo-excitation at $570 \mathrm{~nm}$, we hypothesize that the intensity-dependent behaviors observed in larger quantum wells are promoted by both the smaller binding energies and non-equilibrium effects associated with energy transfer. For example, if fluctuations of the energy gaps for quantum wells $\mathrm{n}=2$ and $\mathrm{n}=3$ induce energy transfer near the isosbestic point between their optical line shapes (near $590 \mathrm{~nm}$ ), then the amount of energy dissipated by the $n=3$ quantum well will be close to $70 \mathrm{meV}$. In this estimate, we have compared the intermediate energy gap of $2.10 \mathrm{eV}(590 \mathrm{~nm})$ to that of the energy acceptor (bandgap at $2.03 \mathrm{eV})$.

\section{Extraction of rate constants from transient absorption signals}

The steady-state emission spectra shown in Fig. 3 suggest that the electronic excitations ultimately concentrate on the bulk-like $\mathrm{n}=\infty$ layer, which is to be expected for an energy funneling process. In this section, the time scales for energy transfer processes are established with transient absorption experiments. The analysis presented in this section corresponds to back-side excitation of the films.

The model used for data analysis is based on the following assumptions. First, it is assumed that electronic excitations in quantum well $n$ transfer only to quantum wells $n-1$ and $n+1$. The rates of the forward and reverse processes are subject to the detailed balance relation. For example, the rate of a transition from quantum well 2 to quantum well 3 is given by $k_{23}=k_{32} \exp \left[\left(E_{3}-E_{2}\right) / k_{B} T\right]$, where $k_{32}$ describes a 

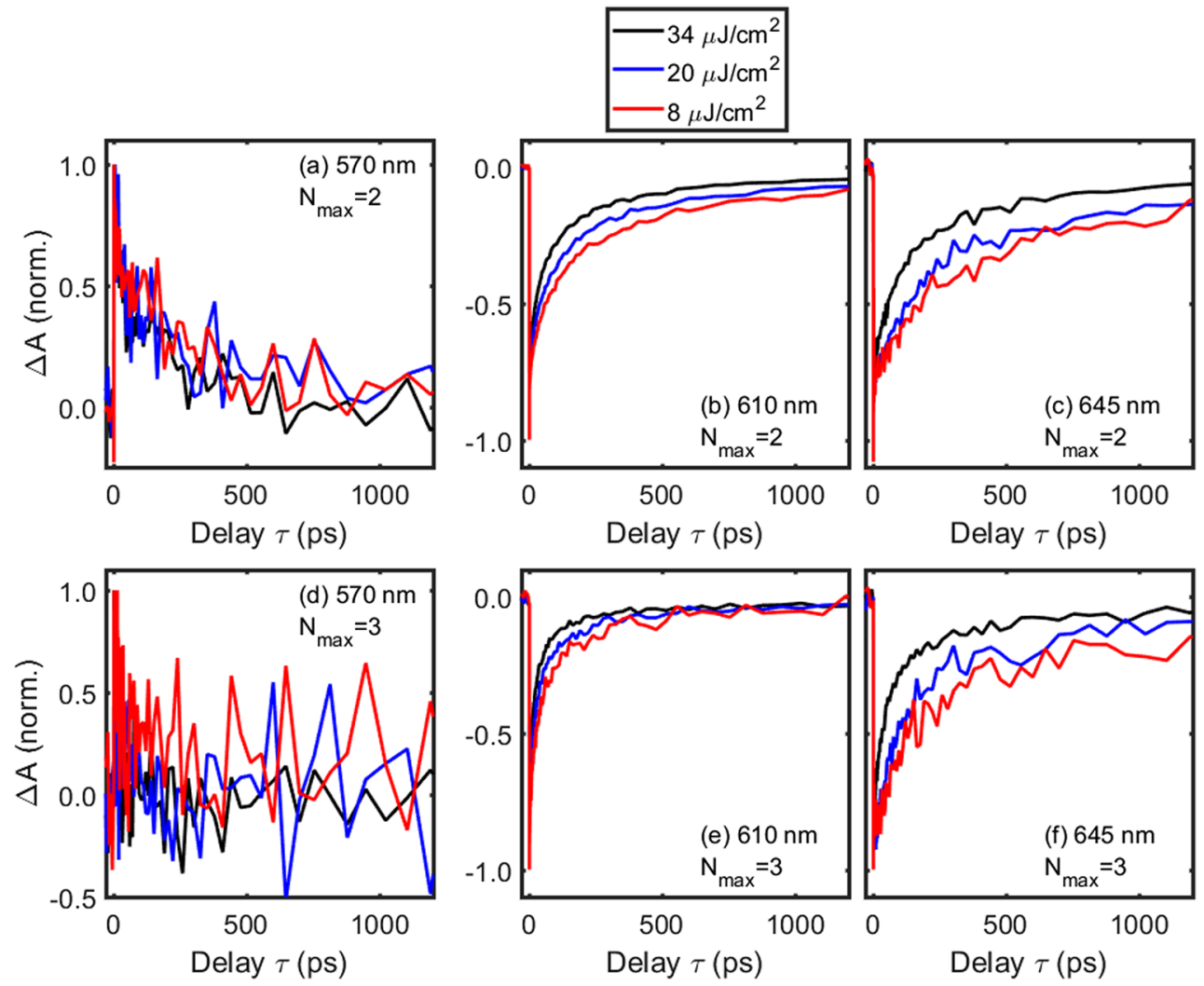

FIG. 7. Transient absorption signals measured for nominal $\mathrm{N}_{\max }=2$ (top) and $\mathrm{N}_{\max }=3$ (bottom) systems with photo-excitation at 610 nm. Detection wavelengths are [(a) and (d)] $570 \mathrm{~nm}$; [(b) and (e)] $610 \mathrm{~nm}$; [(c) and (f)] $645 \mathrm{~nm}$. These wavelengths correspond to the $\mathrm{n}=2,3$, and 4 quantum wells.

transition from quantum well 3 to quantum well 2. Second, we take the signal magnitude to be directly proportional to the population of a particular quantum well. Third, energy transfer between quantum wells is assumed to dominate over electron and/or hole transfer processes because direct optical signatures of such dynamics are not observed within the $500-740 \mathrm{~nm}$ wavelength range at a fluence of $10 \mu \mathrm{J} / \mathrm{cm}^{2} .{ }^{10,11}$ Weak contributions from charge transfer dynamics are also rationalized by the larger exciton binding energies $(>235 \mathrm{meV}$ ) for quantum wells with $\mathrm{n} \leq 5 .^{6}$ Fourth, a process in which excitons migrate to "edge states" is included in the model on the basis of a recent study. ${ }^{6}$ This aspect of the model is motivated by the finding of short $(<100 \mathrm{ps})$ and long-lived $(>1 \mathrm{~ns})$ decay components. Resonances associated with such edge states are not observed in the 500-740 $\mathrm{nm}$ wavelength range.

The set of differential equations used to fit the population dynamics can be written as

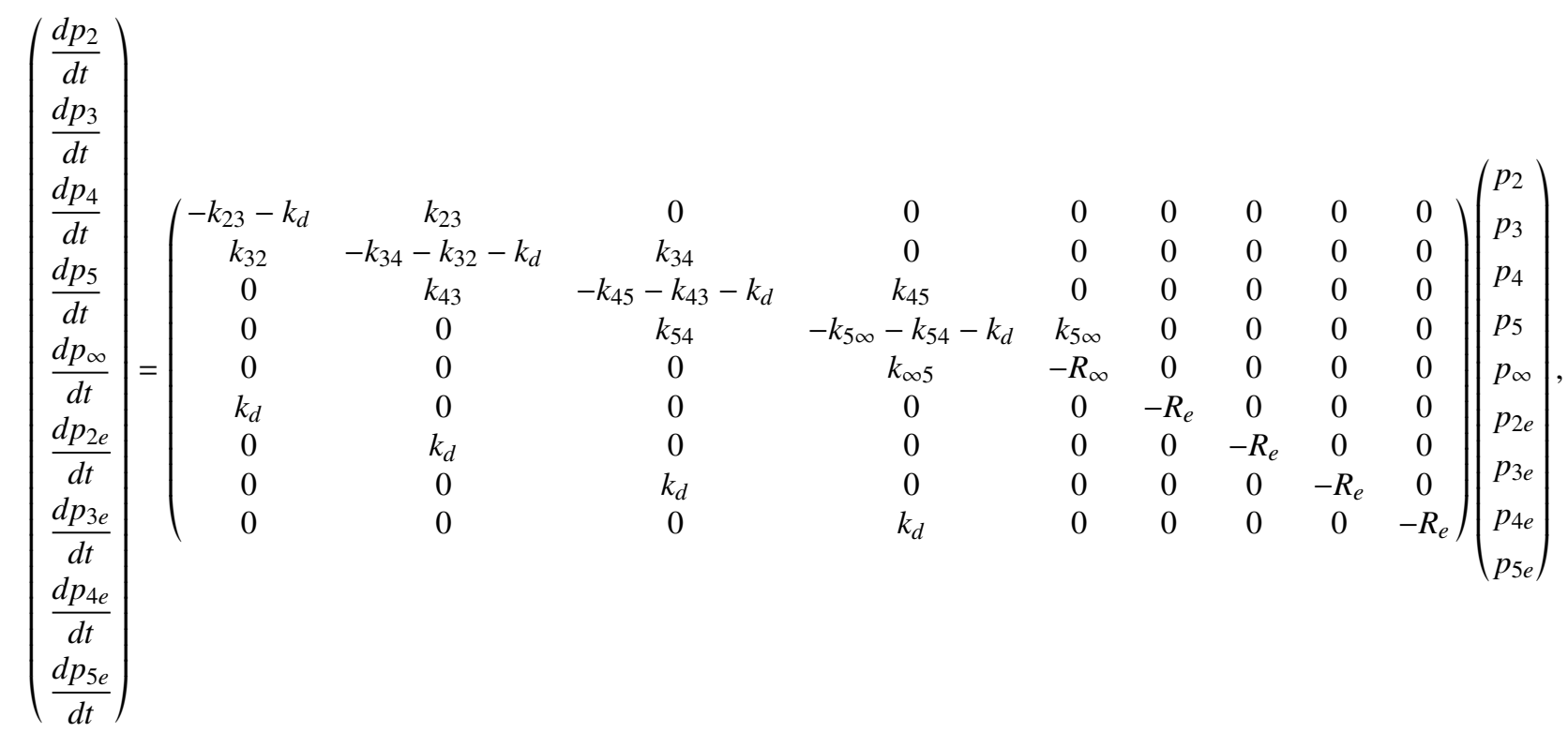


where $p_{n}$ is the population of quantum well $n, p_{n e}$ is the population of an edge state at quantum well $n, R_{\infty}$ is the radiative recombination rate in the $n=\infty$ layer, $R_{e}$ is the defect-assisted recombination rate for all quantum wells, $k_{n m}$ is the rate constant for energy transfer between quantum wells $n$ and $m$, and $k_{d}$ is the rate of exciton diffusion to the edge states. ${ }^{6}$ These parameters are optimized using a least-squares fitting algorithm that simultaneously accounts for transient absorption signals collected between 500 and $740 \mathrm{~nm}$ with the response function given in Appendix A. All fitting parameters are given in Tables I and II.

In Fig. 8, we present fits to transient absorption signals obtained for the $\mathrm{N}_{\max }=2$ system with a pump wavelength of $570 \mathrm{~nm}$ and a fluence of $10 \mu \mathrm{J} / \mathrm{cm}^{2}$. Agreement between the experiment and fit is particularly good at delay times less than $1 \mathrm{~ns}$, whereas discrepancies arise at longer time scales. This indicates that slower decay processes, which are not accounted for in the model, become more prominent at time scales longer than $1 \mathrm{~ns}$. Energy transfer time constants of 115, 170, 90, and $12 \mathrm{ps}$ are found for $k_{23}^{-1}, k_{34}^{-1}, k_{45}^{-1}$, and $k_{5 \infty}^{-1}$, respectively. We note that the time constants, $k_{45}^{-1}$ and $k_{5 \infty}^{-1}$, are not as well constrained as the others because of the weak signal intensity associated with the $n=5$ resonance (i.e., the $n=5$ quantum well has a small concentration). Electron-hole recombination times of 1440 ps are found for the quantum wells $(n=2,3$, 4,5 ), whereas the $n=\infty$ layer possesses a lifetime that is long compared to the delay range. Finally, the time scale of exciton migration to edge states is approximately $160 \mathrm{ps,}$ which is in good agreement with the 200-ps time constant reported in Ref. 6 . Using the rate constants, we compute that an average of $34 \%$ of the excitons dissociate and/or recombine in the $\mathrm{N}_{\max }=2$ system (i.e., average of all quantum wells).

Results similar to those obtained for the $\mathrm{N}_{\max }=2$ system are found by fitting data for the $\mathrm{N}_{\max }=3$ system with the same model (Fig. 9). Again, we employ a pump wavelength of $570 \mathrm{~nm}$ and fluence of $10 \mu \mathrm{J} / \mathrm{cm}^{2}$. The energy transfer time constants are $60,130,31$, and 125 ps for $k_{23}^{-1}, k_{34}^{-1}, k_{45}^{-1}$, and $k_{5 \infty}^{-1}$, respectively. Recombination times are 1290 and 6150 ps for the thin quantum wells $(n=2,3,4,5)$ and $n=\infty$ layer, respectively. The fits generated for the $\mathrm{N}_{\max }=3$ system are quite good throughout the first $1.5 \mathrm{~ns}$, whereas discrepancies arise after $1 \mathrm{~ns}$ in the $\mathrm{N}_{\max }=2$ system. We attribute the superior fits achieved with $\mathrm{N}_{\max }=3$ to the constraints imposed by the intense response of the $n=\infty$ layer in this system. Constraints on the population of the $n=\infty$ layer are particularly important because the $n=\infty$ sub-system is at the bottom of the energy funnel. As in the $\mathrm{N}_{\max }=2$ film, we find that an average of $55 \%$ of excitons dissociate and/or recombine at edge states in the $\mathrm{N}_{\max }=3$ system based on the rate constants. These relaxation processes cause a decrease in signal intensity; however, resonances associated with the free carriers are not detected directly. Therefore, experiments conducted in a broader spectral range will be required to reveal details of the mechanisms.

TABLE I. Parameters used to fit the transient absorption spectrum of $\mathrm{N}_{\max }=2$.

\begin{tabular}{lccc}
\hline \hline Parameter ${ }^{\mathrm{a}, \mathrm{b}, \mathrm{c}, \mathrm{d}}$ & Value & Parameter $\mathrm{b}, \mathrm{c}, \mathrm{d}$ & \\
\hline$k_{23}^{-1}$ & $115 \pm 3 \mathrm{ps}$ & $\omega_{e g, 2}$ & $575 \mathrm{~nm}(2.16 \mathrm{eV})$ \\
$k_{34}^{-1}$ & $169 \pm 8 \mathrm{ps}$ & $\omega_{e g, 3}$ & $614 \mathrm{~nm}(2.02 \mathrm{eV})$ \\
$k_{45}^{-1}$ & $91 \pm 13 \mathrm{ps}$ & $\omega_{e g, 4}$ & $648 \mathrm{~nm}(1.91 \mathrm{eV})$ \\
$k_{5 \infty}^{-1}$ & $12 \pm 4 \mathrm{ps}$ & $\omega_{e g, 5}$ & $673 \mathrm{~nm}(1.84 \mathrm{eV})$ \\
$k_{d}^{-1}$ & $161 \pm 6 \mathrm{ps}$ & $\omega_{e g, \infty}$ & $750 \mathrm{~nm}(1.65 \mathrm{eV})$ \\
$R_{e}$ & $1439 \pm 38 \mathrm{ps}$ & $\omega_{f e, 2}$ & $547 \mathrm{~nm}(2.27 \mathrm{eV})$ \\
$R_{\infty}$ & $53036 \pm 43885 \mathrm{ps}$ & $\omega_{f e, 3}$ & $584 \mathrm{~nm}(2.12 \mathrm{eV})$ \\
$p_{2}^{0}$ & $1.71 \pm 13.73$ & $\omega_{f e, 4}$ & $624 \mathrm{~nm}(1.99 \mathrm{eV})$ \\
$p_{3}^{0}$ & $1.07 \pm 8.59$ & $\omega_{f e, 5}$ & $670 \mathrm{~nm}(1.85 \mathrm{eV})$ \\
$p_{4}^{0}$ & $0.45 \pm 3.58$ & $\omega_{f e, \infty}$ & $610 \mathrm{~nm}(2.03 \mathrm{eV})$ \\
$p_{5}^{0}$ & $0.05 \pm 0.37$ & $\Delta_{e g, 2}$ & $17 \pm 1 \mathrm{~nm}(0.06 \pm 0.003 \mathrm{eV})$ \\
$p_{\infty}^{0}$ & $0.00 \pm 0.02$ & $\Delta_{e g, 3}$ & $17 \pm 1 \mathrm{~nm}(0.06 \pm 0.002 \mathrm{eV})$ \\
$\mu_{e g, 2}$ & $0.96 \pm 1.93$ & $\Delta_{e g, 4}$ & $19 \pm 2 \mathrm{~nm}(0.06 \pm 0.006 \mathrm{eV})$ \\
$\mu_{e g, 3}$ & $1.10 \pm 2.20$ & $\Delta_{e g, 5}$ & $32 \pm 7 \mathrm{~nm}(0.09 \pm 0.020 \mathrm{eV})$ \\
$\mu_{e g, 4}$ & $0.98 \pm 1.97$ & $\Delta_{e g, \infty}$ & $141 \pm 500 \mathrm{~nm}(0.31 \pm 1.110 \mathrm{eV})$ \\
$\mu_{e g, 5}$ & $1.98 \pm 4.05$ & $\Delta_{f e, 2}$ & $21 \pm 1 \mathrm{~nm}(0.09 \pm 0.005 \mathrm{eV})$ \\
$\mu_{e g, \infty}$ & $\Delta_{f e, 3}$ & $16 \pm 1 \mathrm{~nm}(0.06 \pm 0.004 \mathrm{eV})$ \\
$\mu_{f e, 2}$ & $\Delta_{f e, 4}$ & $15 \pm 2 \mathrm{~nm}(0.05 \pm 0.007 \mathrm{eV})$ \\
$\mu_{f e, 3}$ & $1.28 \pm 0.57$ & $\Delta_{f e, 5}$ & $13 \pm 7 \mathrm{~nm}(0.04 \pm 0.023 \mathrm{eV})$ \\
$\mu_{f e, 4}$ & $1.23 \pm 2.48$ & $\Delta_{f e, \infty}$ & $132 \mathrm{~nm}(0.43 \mathrm{eV})$ \\
$\mu_{f e, 5}$ & $1.23 \pm 2.48$ & & \\
$\mu_{f e, \infty}$ & $2.19 \pm 4.48$ & & \\
\hline
\end{tabular}

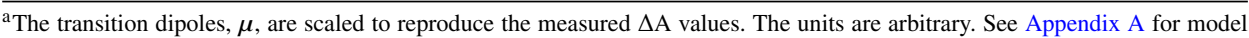
equations.

${ }^{b}$ Fits are conducted in the frequency domain under the assumption of Gaussian line shapes.

${ }^{\mathrm{c}}$ The single exciton and biexciton resonance frequencies were fixed to the peak values during the fits. The frequencies were first optimized at multiple delay times, which showed that these parameters are delay-independent.

${ }^{\mathrm{d}}$ Error ranges represent $95 \%$ confidence intervals. 
TABLE II. Parameters used to fit the transient absorption signal for $\mathrm{N}_{\max }=3$.

\begin{tabular}{|c|c|c|c|}
\hline Parameter ${ }^{\mathrm{a}, \mathrm{b}, \mathrm{c}, \mathrm{d}}$ & Value & Parameter $^{\mathrm{b}, \mathrm{c}, \mathrm{d}}$ & Value \\
\hline$k_{23}^{-1}$ & $61 \pm 2 \mathrm{ps}$ & $\omega_{e g, 2}$ & $573 \mathrm{~nm}(2.16 \mathrm{eV})$ \\
\hline$k_{34}^{-1}$ & $130 \pm 9 \mathrm{ps}$ & $\omega_{e g, 3}$ & $616 \mathrm{~nm}(2.01 \mathrm{eV})$ \\
\hline$k_{45}^{-1}$ & $32 \pm 10 \mathrm{ps}$ & $\omega_{e g, 4}$ & $649 \mathrm{~nm}(1.91 \mathrm{eV})$ \\
\hline$k_{5 \infty}^{-1}$ & $127 \pm 78 \mathrm{ps}$ & $\omega_{e g, 5}$ & $676 \mathrm{~nm}(1.83 \mathrm{eV})$ \\
\hline$k_{d}^{-1}$ & $61 \pm 2 \mathrm{ps}$ & $\omega_{e g, \infty}$ & $750 \mathrm{~nm}(1.65 \mathrm{eV})$ \\
\hline$R_{e}$ & $1288 \pm 19 \mathrm{ps}$ & $\omega_{f e, 2}$ & $551 \mathrm{~nm}(2.25 \mathrm{eV})$ \\
\hline$R_{\infty}$ & $6150 \pm 253 \mathrm{ps}$ & $\omega_{f e, 3}$ & $586 \mathrm{~nm}(2.12 \mathrm{eV})$ \\
\hline$p_{2}^{0}$ & $0.64 \pm 0.40$ & $\omega_{f e, 4}$ & $630 \mathrm{~nm}(1.97 \mathrm{eV})$ \\
\hline$p_{3}^{0}$ & $0.61 \pm 0.38$ & $\omega_{f e, 5}$ & $657 \mathrm{~nm}(1.89 \mathrm{eV})$ \\
\hline$p_{4}^{0}$ & $0.17 \pm 0.12$ & $\omega_{f e, \infty}$ & $610 \mathrm{~nm}(2.03 \mathrm{eV})$ \\
\hline$p_{5}^{0}$ & $0.59 \pm 0.99$ & $\Delta_{e g, 2}$ & $15 \pm 1 \mathrm{~nm}(0.06 \pm 0.004 \mathrm{eV})$ \\
\hline$p_{\infty}^{0}$ & $0.80 \pm 0.51$ & $\Delta_{e g, 3}$ & $17 \pm 0 \mathrm{~nm}(0.06 \pm 0.002 \mathrm{eV})$ \\
\hline$\mu_{e g, 2}$ & $1.17 \pm 0.18$ & $\Delta_{e g, 4}$ & $21 \pm 2 \mathrm{~nm}(0.06 \pm 0.007 \mathrm{eV})$ \\
\hline$\mu_{e g, 3}$ & $1.19 \pm 0.19$ & $\Delta_{e g, 5}$ & $14 \pm 2 \mathrm{~nm}(0.04 \pm 0.005 \mathrm{eV})$ \\
\hline$\mu_{e g, 4}$ & $1.83 \pm 0.40$ & $\Delta_{e g, \infty}$ & $142 \pm 9 \mathrm{~nm}(0.31 \pm 0.020 \mathrm{eV})$ \\
\hline$\mu_{e g, 5}$ & $0.57 \pm 0.46$ & $\Delta_{f e, 2}$ & $27 \pm 5 \mathrm{~nm}(0.11 \pm 0.020 \mathrm{eV})$ \\
\hline$\mu_{e g, \infty}$ & $0.95 \pm 0.16$ & $\Delta_{f e, 3}$ & $18 \pm 1 \mathrm{~nm}(0.07 \pm 0.005 \mathrm{eV})$ \\
\hline$\mu_{f e, 2}$ & $0.72 \pm 0.11$ & $\Delta_{f e, 4}$ & $16 \pm 3 \mathrm{~nm}(0.05 \pm 0.010 \mathrm{eV})$ \\
\hline$\mu_{f e, 3}$ & $1.01 \pm 0.16$ & $\Delta_{f e, 5}$ & $10 \pm 5 \mathrm{~nm}(0.03 \pm 0.015 \mathrm{eV})$ \\
\hline$\mu_{f e, 4}$ & $1.72 \pm 0.37$ & $\Delta_{f e, \infty}$ & $132 \mathrm{~nm}(0.43 \mathrm{eV}) \pm 0 \%$ \\
\hline$\mu_{f e, 5}$ & $0.85 \pm 0.68$ & & \\
\hline$\mu_{f e, \infty}$ & $0.63 \pm 0.12$ & & \\
\hline
\end{tabular}

${ }^{\mathrm{a}}$ The transition dipoles, $\mu$, are scaled to reproduce the measured $\Delta \mathrm{A}$ values. The units are arbitrary. See Appendix A for model equations.

${ }^{b}$ Fits are conducted in the frequency domain under the assumption of Gaussian line shapes.

${ }^{c}$ The single exciton and biexciton resonance frequencies were fixed to the peak values during the fits. The frequencies were first optimized at multiple delay times, which showed that these parameters are delay-independent.

${ }^{\mathrm{d}}$ Error ranges represent $95 \%$ confidence intervals.

\section{E. 2DTA signatures of energy transfer dynamics}

In this section, we present 2DTA spectra acquired for the $\mathrm{N}_{\max }=2$ and $\mathrm{N}_{\max }=3$ systems. Like 2D photon-echo spectra, the present 2DTA spectra can be interpreted by decomposing the signal into diagonal and off-diagonal contributions..$^{22,23,25}$
Peaks on the diagonal of the spectra primarily represent the GSB signal component of the ground electronic state, whereas off-diagonal peaks carry information about non-radiative transitions between quantum wells. This clear delineation between diagonal and off-diagonal signal components is not exhibited by strongly coupled quantum systems in which the GSB
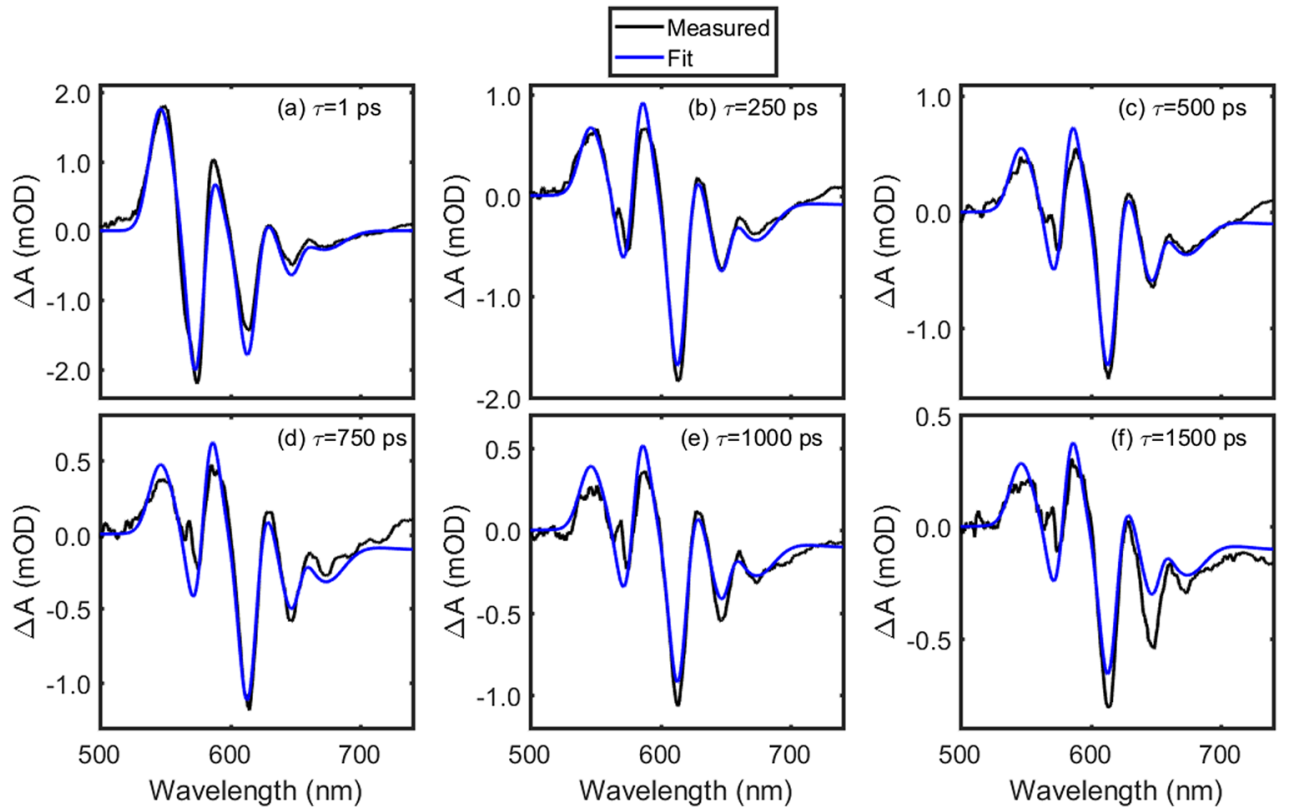

FIG. 8. Transient absorption signals measured (black) and calculated (blue) for the $\mathrm{N}_{\max }=2$ system. The laser fluence is $10 \mu \mathrm{J} / \mathrm{cm}^{2}$. Model calculations are conducted with the model outlined in Appendix A and the parameters given in Table I. 


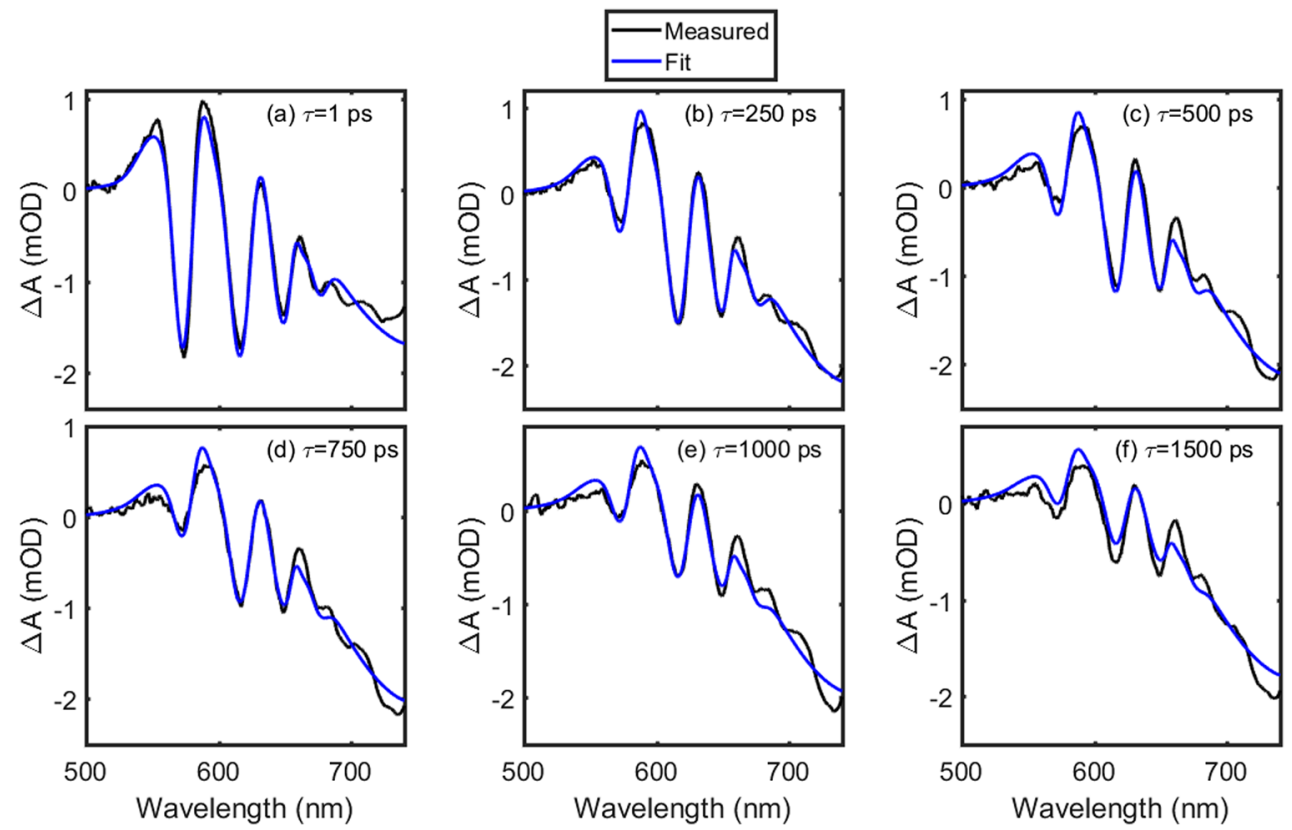

FIG. 9. Transient absorption signals measured (black) and calculated (blue) for the $\mathrm{N}_{\max }=3$ system. The laser fluence is $10 \mu \mathrm{J} / \mathrm{cm}^{2}$. Model calculations are conducted with the model outlined in Appendix A and the parameters given in Table II.

nonlinearity possesses intense off-diagonal terms..$^{49,50}$ For perturbatively coupled quantum wells, growth in the intensity of off-diagonal peaks (i.e., the ESE signal component) is a signature of energy transfer between quantum wells. From this perspective, the pattern of 2DTA resonances found in the layered perovskite system is analogous to that of a molecular aggregate or photosynthetic protein in which the intermolecular couplings are not sufficient to promote Frenkel exciton delocalization. ${ }^{50}$

Spectroscopic signatures of strong coupling deserve further discussion as inter-quantum well biexciton states were recently reported for a related layered 2D perovskite system. $^{51}$ It is instructive to first consider the physical parameters that promote delocalization. To a good approximation, electrons are not shared between the perovskite quantum wells and the coupling is Coulombic in nature. ${ }^{52,53}$ Therefore, the delocalization of excitons between quantum wells may take hold when the Coulombic coupling is comparable to or larger than the difference in bandgaps. For example, the difference in bandgaps determined above for the $\mathrm{n}=2$ and $\mathrm{n}=3$ quantum wells is approximately $150 \mathrm{meV}$, whereas the Coulombic coupling between transition dipoles is approximately $9.3 \mathrm{meV}$ (see Appendix B). The mixing angle associated with this pair of quantum wells is given by

$$
\theta=\frac{1}{2} \operatorname{atan}\left(\frac{2 J}{\Delta E}\right)
$$

where $J$ is the Coulombic coupling and $\Delta E$ is the difference in bandgaps. With these parameters, the excitons are roughly 99\% localized on the individual $\mathrm{n}=2$ and $\mathrm{n}=3$ quantum wells.

The differences between GSB responses found in the limits of strong and weak (i.e., perturbative) couplings are illustrated in Fig. 10. In the strong coupling limit, the offdiagonal peaks have intensities that are approximately equal to those of the diagonal peaks when all transition dipoles have similar magnitudes. ${ }^{49}$ It is instructive to consider that
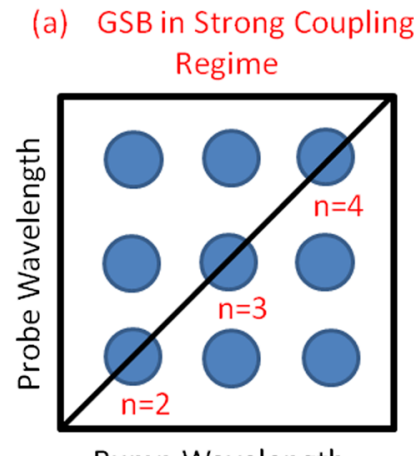

Pump Wavelength (b) GSB in Weak Coupling Regime

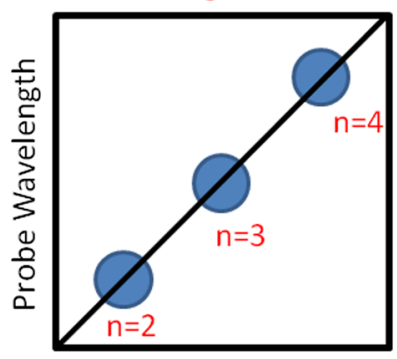

Pump Wavelength (c) ESE in Weak Coupling Regime

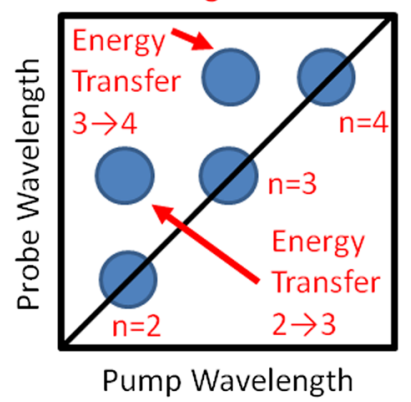

FIG. 10. (a) The GSB response exhibits intense off-diagonal peaks in the strong-coupling regime. (b) Off-diagonal peaks do not contribute to the GSB signal component with perturbative Coulombic coupling. (c) In the weak-coupling regime, off-diagonal peaks with negative signs above the diagonal arise from the ESE signal component and signify energy transfer transitions. 
the GSB response function involves two nested summations over exciton states in the strong coupling regime (i.e., diagonal and off-diagonal terms appear). ${ }^{54}$ By contrast, the diagonal peaks dominate in the weak coupling limit because the GSB response function can be written as a sum over a single exciton index [see Eqs. (A1) and (A2)]. ${ }^{50}$ For these reasons, the 2DTA results obtained for the present system are most consistent with perturbative energy transfer as opposed to a mechanism involving delocalized states.

Diagonal peaks with negative signs, which represent the sum of GSB and ESE responses, dominate the response of the $\mathrm{N}_{\max }=2$ system at a delay time of 1 ps in Fig. 11. Offdiagonal peaks with negative signs (i.e., the ESE response) correlate the initially populated quantum well to the quantum well in which the excitation resides at time, $\tau$. For example, at a delay time of $1 \mathrm{~ns}$, the most intense cross peak associated with 570-nm excitation is found with 610-nm detection, which represents a transition between the $\mathrm{n}=2$ and $\mathrm{n}=3$ quantum wells. Similarly, the prominent cross peak associated with 610-nm excitation and 645-nm detection represents a transition between the $n=3$ and $n=4$ quantum wells. At delay times less than $1 \mathrm{~ns}$, the weak signal strength found with 570-nm excitation and 645-nm detection suggests that the $n=2$ quantum well transfers an electronic excitation directly to the $n=3$ quantum well rather than to $n=4$. Thus, the patterns of resonances further support the assumptions associated with the kinetic model employed in Sec. III D. Signal emission at wavelengths longer than $700 \mathrm{~nm}$, which is associated with the $n=\infty$ layer, is below the noise floor of the experiment for the $\mathrm{N}_{\max }=2$ system (i.e., the $\mathrm{n}=\infty$ concentration is low).
In Fig. 12, measured and calculated 2DTA spectra are presented for the $\mathrm{N}_{\max }=3$ system. As in Fig. 11, good agreement between experiment and theory is found at wavelengths shorter than $700 \mathrm{~nm}$, where the excitons are most tightly bound. Like the $\mathrm{N}_{\max }=2$ system, the pattern of resonances is consistent with a physical picture in which quantum well $\mathrm{n}$ primarily transfers energy to quantum well $\mathrm{n}+1$ (i.e., an energetically "downhill" process). In the simulated 2DTA spectra, the signal intensity is underestimated at pump wavelengths less than $650 \mathrm{~nm}$ and probe wavelengths greater than $700 \mathrm{~nm}$. This discrepancy becomes apparent in less than 100 ps, which is fast compared to the cascade of energy transfer events. Therefore, rather than assigning these features to ultrafast interquantum well transitions, we attribute this difference to pump absorption at wavelengths less than $650 \mathrm{~nm}$ by the $n=\infty$ layer. The issue is that the model incorporates excitons for each quantum well but does not include the full manifold of higher-lying states for the $n=\infty$ layer. Therefore, photoinitiated carrier cooling processes in which the $n=\infty$ layer absorbs light far above the bandgap are not accounted for. These cooling processes weakly influence energy transfer dynamics because of the low probability of the $n=\infty$ layer transferring electronic excitations "uphill" to smaller quantum wells.

The 2DTA spectra simulated in Figs. 11 and 12 employ the parameters obtained in Sec. III D, which are based on experiments conducted with a 570-nm pump laser. All parameters apply to 2DTA as well; however, the initial conditions depend on the pump wavelength. For this reason, these initial populations are estimated based on the linear absorbance spectrum and the transient absorption signal intensity at short
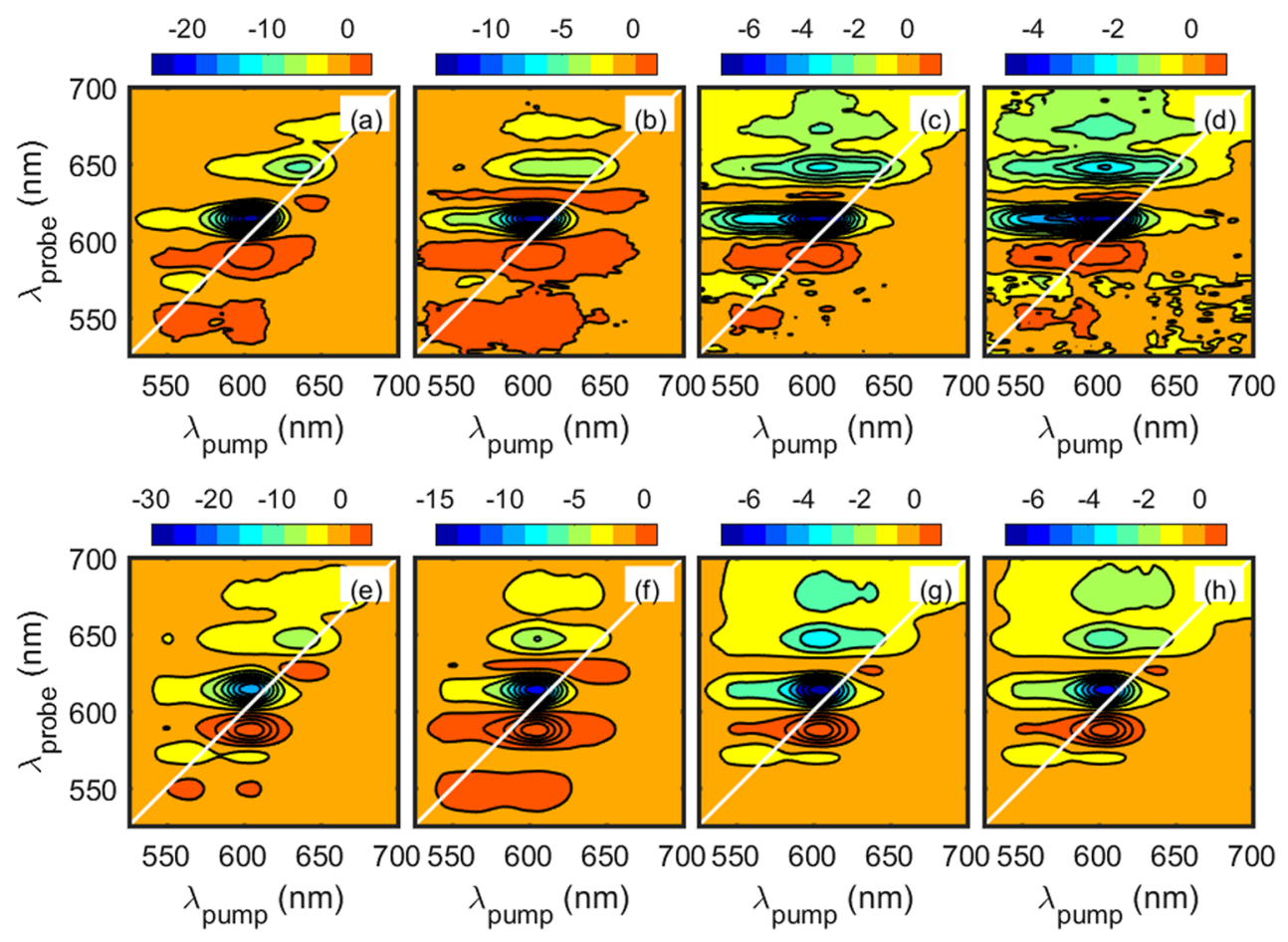

FIG. 11. Measured (top) and calculated (bottom) 2DTA spectra of $\mathrm{N}_{\max }=2$ system acquired with back-side excitation. Delay times are [(a) and (e)] 1 ps; [(b) and (f)] $100 \mathrm{ps}$; [(c) and (g)] $500 \mathrm{ps;} \mathrm{[(d)} \mathrm{and} \mathrm{(h)]} 1000$ ps. Cross peaks above the diagonal represent energy transfer between quantum wells with different $\mathrm{n}$ values. The 2DTA spectra are calculated using the model outlined in Appendix A and the parameters in Table I. In the simulated 2DTA spectra, the frequency variables are related to the spectral axes by $\lambda_{\text {pump }}=2 \pi c / \omega_{p u}$ and $\lambda_{\text {probe }}=2 \pi c / \omega_{p r}$. 

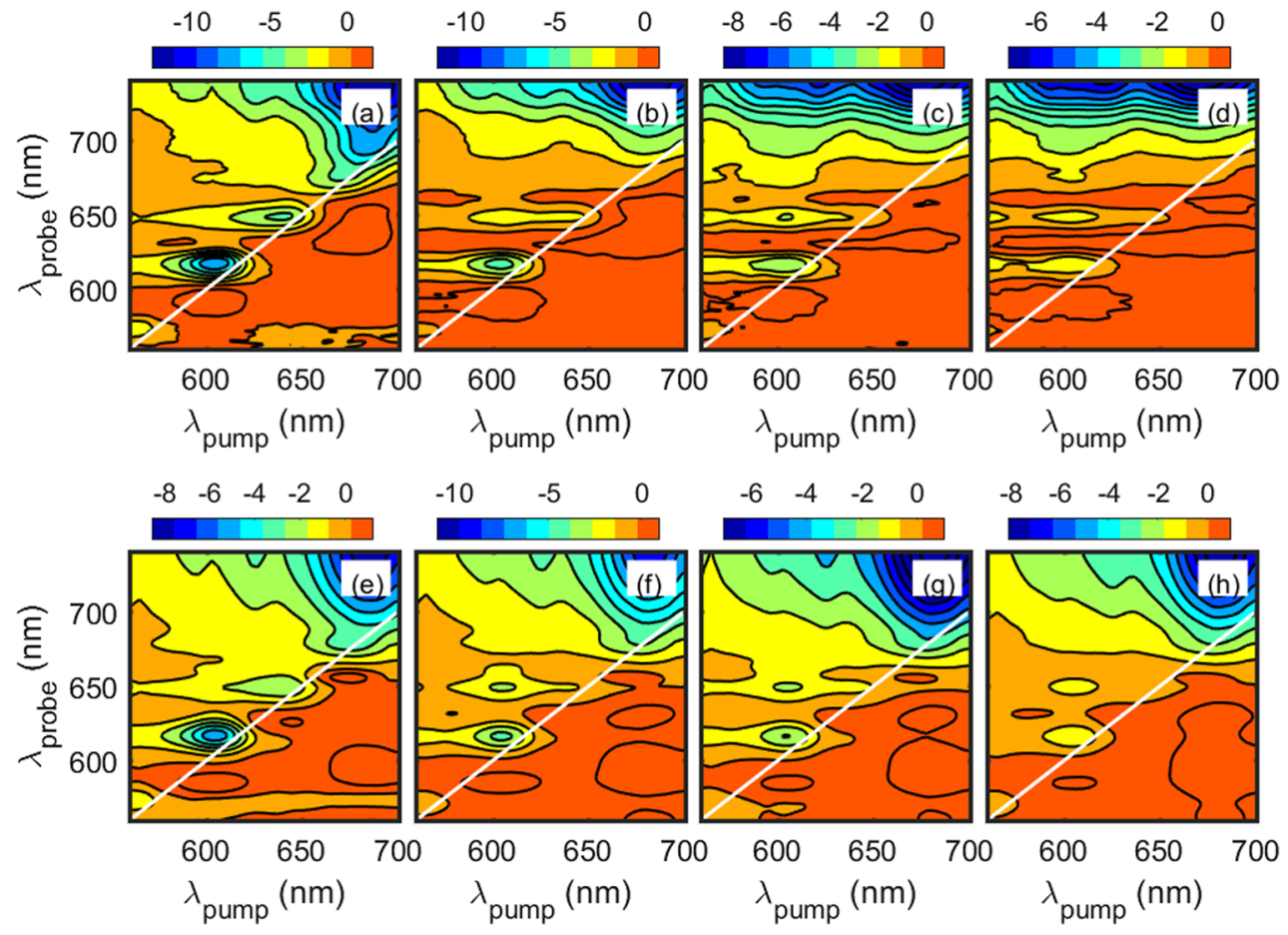

FIG. 12. Measured (top) and calculated (bottom) 2DTA spectra of $\mathrm{N}_{\max }=3$ system acquired with back-side excitation. Delay times are [(a) and (e)] 1 ps; [(b) and (f)] $100 \mathrm{ps;} \mathrm{[(c)} \mathrm{and} \mathrm{(g)]} 500$ ps; [(d) and (h)] 1000 ps. Cross peaks above the diagonal represent energy transfer between quantum wells with different values of $\mathrm{n}$. The 2DTA spectra are calculated using the model outlined in Appendix A and the parameters in Table II. In the simulated 2DTA spectra, the frequency variables in the Appendix are related to the spectral axes by $\lambda_{\text {pump }}=2 \pi c / \omega_{p u}$ and $\lambda_{\text {probe }}=2 \pi c / \omega_{p r}$.

delay times in the simulated 2DTA spectra (i.e., before energy transfer takes hold).

The 2DTA presented in this section confirms the following points. First, the 2DTA spectra for both systems do not exhibit off-diagonal GSB responses, which would be evident at a delay time of 1 ps (i.e., before energy transfer redistributes signal intensity). This aspect of the 2DTA spectra suggests perturbative Coulombic coupling between quantum wells (i.e., regime of energy transfer as opposed to exciton delocalization). Second, the 2DTA spectra further indicate that the energy transfer dynamics are dominated by transitions between quantum wells with indices that differ by 1 . For example, cross peaks between the $n=2$ and $n=3$ quantum wells appear before those involving the $n=2$ and $n=4$ quantum wells in both systems, thereby suggesting sequential energy transfer processes. Slow energy transfer dynamics between quantum wells 2 and 4 (or 3 and 5, etc.) are consistent with poor donor-acceptor spectral overlap in the Förster mechanism (see Appendix B).

\section{SUMMARY OF PHOTO-INDUCED RELAXATION PROCESSES}

The relaxation scheme proposed in this work is summarized in Fig. 13. Our analysis suggests that light absorption by the $n=2$ quantum well initiates a cascade of energy transfer processes in the direction of increasing $n$ (i.e., towards the front of the film). Because of the spectral overlap factor in Förster's rate formula [see Eq. (B5)], these energy transfer transitions occur sequentially between quantum wells whose indices differ by 1 . Electronic excitations are funneled to the $n=\infty$ layer within $1 \mathrm{~ns}$, where they relax through radiative recombination. In addition, we find that a separate non-radiative relaxation process must be introduced to fit the transient absorption data. Evidence for this process is indirect in the sense that it induces a decay in the signal magnitude but does not yield additional resonances in the visible spectral range. We assign these "dark" decay components to diffusion and non-radiative recombination at edge states based on the recent literature for a similar system. ${ }^{6}$ Of course, it is possible that numerous non-radiative decay channels such as exciton dissociation and/or charge transfer contribute to the decay in the signal magnitude as well. Because resonances associated with these decay channels are not observed, experiments conducted in a wider spectral range may be required to assign these parallel processes without ambiguity.

Most importantly, we conclude that the spectroscopic signatures observed in the visible spectral range signify energy transfer dynamics rather than electron and/or hole transport. First, the data are well described with the basis set of bound single excitons and biexcitons (see Fig. 4). Second, the frequencies and linewidths of the single exciton resonances in the transient absorption signals are quite similar to those exhibited by the linear absorbance spectrum, which should be expected for an excitonic response. Third, the data clearly indicate sequential energy transfer processes between quantum wells whose indices differ by 1 , which is consistent with the constraint imposed by the donor-acceptor spectral overlap in Förster's rate formula (see Appendix B). Fourth, contributions from energy transfer dynamics should be anticipated for these systems based on the large transition dipoles and relatively short distances $(0.9 \mathrm{~nm})$ between neighboring 


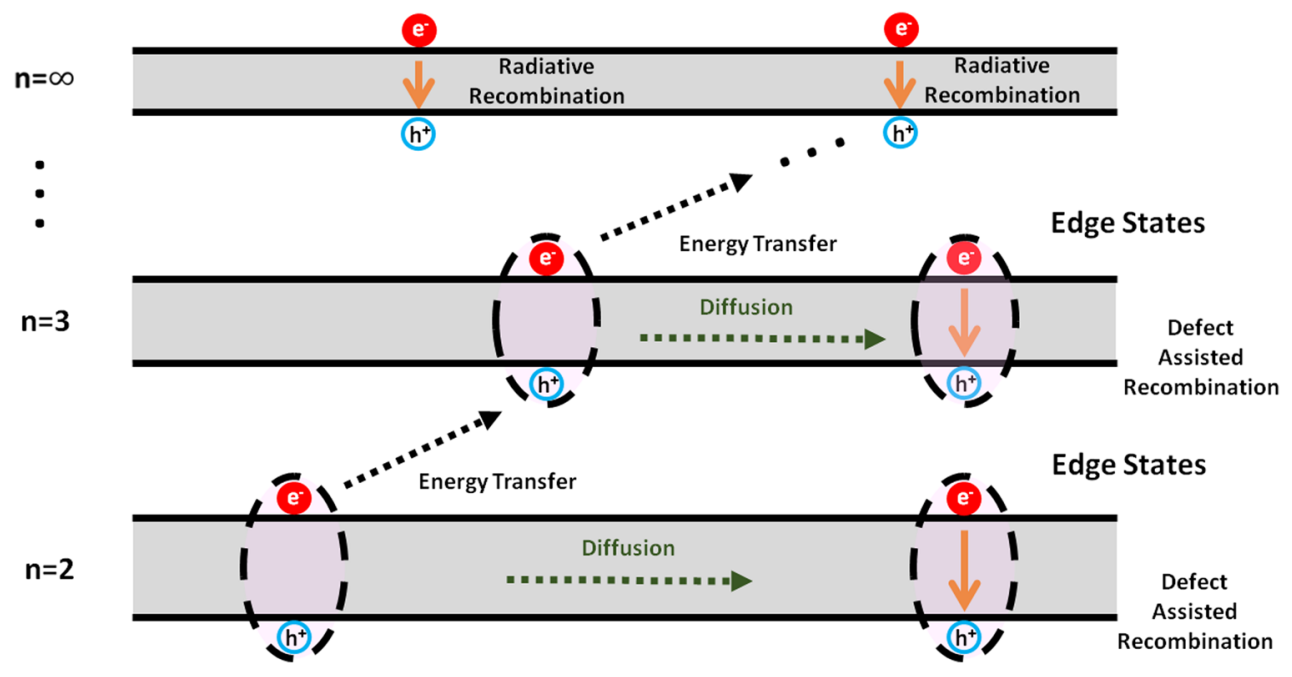

FIG. 13. Relaxation scheme for layered perovskite quantum wells. Energy transfer dynamics dominate the spectroscopic signals. Additional non-radiative decay pathways (e.g., radiative and defect-assisted recombination) are incorporated into the model based on fits to the transient absorption data. Time constants associated with these processes are given in Tables I and II.

quantum wells. As shown in Appendix B, time constants on the order of 100 ps are predicted by Förster's rate formula. Fifth, the dipole-dipole coupling that drives energy transfer scales as the inverse of the distance between the donor and acceptor cubed [see Eq. (B2)], whereas the coupling associated with charge transfer decays exponentially and will therefore be competitive only at shorter distances.

Overall, it appears that the present systems are ideally suited for funneling electronic excitations to the $n=\infty$ layer on the sub-ns time scale. The $\mathrm{n}=\infty$ layer then relaxes primarily by way of radiative recombination. Electron and hole transfer may contribute at longer delay times but will be outcompeted by energy transfer on the sub-500-ps time scale.

\section{CONCLUDING REMARKS}

In summary, transient absorption experiments and model calculations have been used to reveal an energy funneling mechanism in the present layered 2D perovskites. Following light absorption, electronic excitations are sequentially transferred between quantum wells in the direction of increasing $\mathrm{n}$ on the sub-ns time scale (see Fig. 13). This cascade of energy transfer processes is both energetically and spatially directed because the average value of $\mathrm{n}$ increases from the back to the front of the film. We additionally find that secondary non-radiative decay channels must be incorporated to fit the transient absorption data. These relaxation processes are assigned to non-radiative recombination at edge states based on the recent literature, ${ }^{6}$ however, this conclusion is tentative because the spectroscopic signatures are indirect (i.e., resonances associated with edge states are not observed in the transient absorption). Evidence for electron and/or hole transfer dynamics is not observed in these systems but this does not necessarily rule out such dynamics at longer time scales. For example, it is possible that electron and/or hole transfer dynamics do not yield intense resonances in the visible spectral range. Experiments conducted at longer delay times with more widely tunable probe pulses may be required to assign the dynamics that occur subsequent to energy funneling into the $n=\infty$ layer.

\section{SUPPLEMENTARY MATERIAL}

See supplementary material for experimental signatures of many-body processes, 2DTA spectra acquired with frontside excitation, approximations needed to obtain Gaussian line shapes in model calculations, 2D orientational averages, SEM images of layered 2D perovskites, and X-ray diffraction data.

\section{ACKNOWLEDGMENTS}

This work is supported by the National Science Foundation under Grant Nos. CHE-1504350 (Z.G. and A.M.) and DMR-1610879 (J.H. and W.Y.). We thank Thomas Cheshire for evaluating the orientationally averaged dipole-dipole couplings given in the supplementary material. SEM measurements were performed at the Chapel Hill Analytical and Nanofabrication Laboratory, CHANL, a member of the North Carolina Research Triangle Nanotechnology Network, RTNN, which is supported by the National Science Foundation, Grant No. ECCS-1542015, as part of the National Nanotechnology Coordinated Infrastructure, NNCI. PXRD measurements were conducted at the Analytical Instrumentation Facility (AIF) at North Carolina State University, which is supported by the State of North Carolina and the National Science Foundation (Award No. ECCS-1542015). The AIF is a member of the North Carolina Triangle Nanotechnology Network (RTNN), a site in the National Nanotechnology Coordinated Infrastructure (NNCI).

\section{APPENDIX A: RESPONSE FUNCTIONS FOR TRANSIENT ABSORPTION SPECTROSCOPY}

The transient absorption response (i.e., third-order perturbative optical response) is described with the six double-sided Feynman diagrams shown in Fig. 14. ${ }^{22,23,54}$ Here, we have 

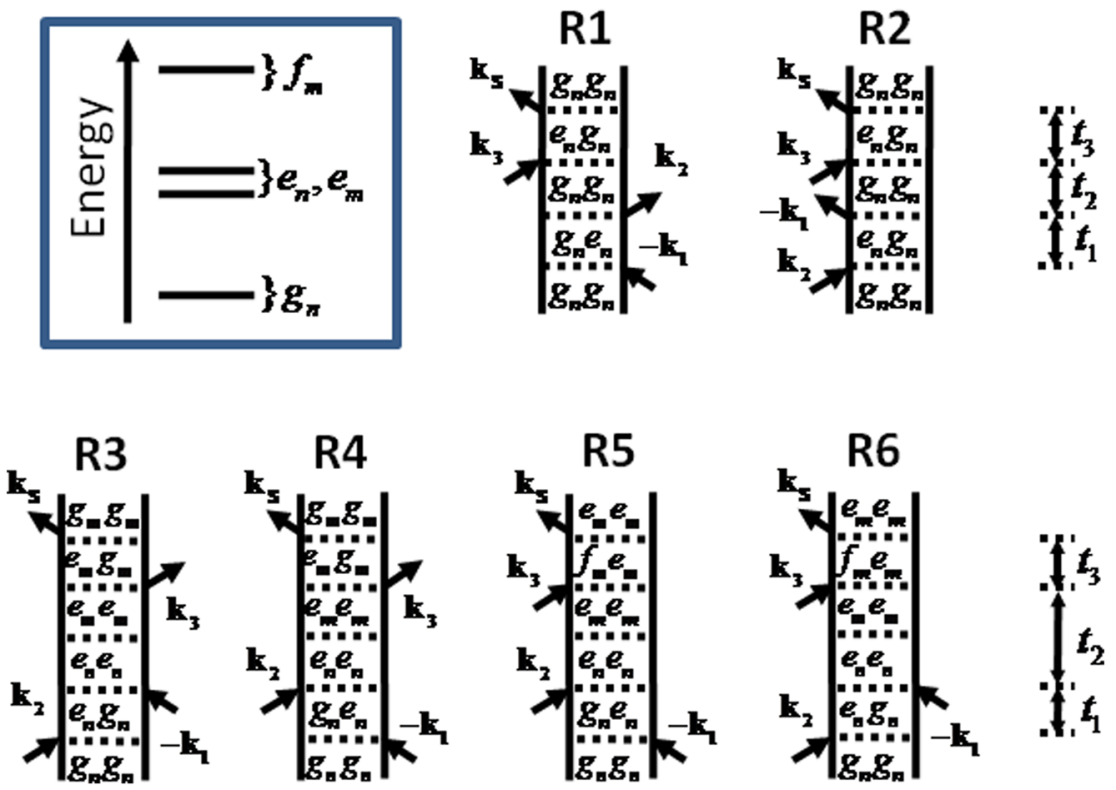

FIG. 14. Double-sided Feynman diagrams for transient absorption response. Electronic states are labeled according to the notation given in Fig. 4. The resonance frequency for single exciton transitions is $\omega_{e g, n}$, whereas $\omega_{f e, n}$ corresponds to biexciton transitions. The subscript, $n$, denotes the particular quantum well. introduced the possibility of population transfer in the $t_{2}$ interval between field-matter interactions, which corresponds to the pump-probe delay time.

The GSB signal components are given by

$$
\begin{aligned}
R_{1}^{(3)}\left(\omega_{p u}, \tau, \omega_{p r}\right)= & -\sum_{n} p_{n}^{0} p_{n}(\tau)\left|\mu_{e_{n} g_{n}}\right|^{4} \Phi_{g_{n} e_{n}}\left(\omega_{p u}\right) \\
& \times \Phi_{e_{n} g_{n}}\left(\omega_{p r}\right)
\end{aligned}
$$

and

$$
\begin{aligned}
R_{2}^{(3)}\left(\omega_{p u}, \tau, \omega_{p r}\right)= & -\sum_{n} p_{n}^{0} p_{n}(\tau)\left|\mu_{e_{n} g_{n}}\right|^{4} \Phi_{e_{n} g_{n}}\left(\omega_{p u}\right) \\
& \times \Phi_{e_{n} g_{n}}\left(\omega_{p r}\right),
\end{aligned}
$$

where $\mu_{e x, n}$ is the transition dipole for single exciton resonance in quantum well $n, \omega_{p u}$ is the pump frequency, $\omega_{p r}$ is the probe frequency, and $\Phi_{e x, n}(\omega)$ is a Gaussian line shape,

$$
\Phi_{e x, n}(\omega)=\exp \left[-4 \ln (2) \frac{\left(\omega-\omega_{e x, n}\right)^{2}}{\Delta_{e x, n}^{2}}\right] .
$$

Spectroscopic line shapes are usually taken to be Lorentzian when the signal is written as a product of one-dimensional functions. $^{22}$ The assumptions needed to support Gaussian line shapes, which are motivated by agreement with the experimental measurements, are outlined in the supplementary material. The ESE signal components are written as

$$
\begin{aligned}
R_{3}^{(3)}\left(\omega_{p u}, \tau, \omega_{p r}\right)= & -\sum_{n m} p_{n}^{0} p_{m}(\tau)\left|\mu_{e_{n} g_{n}}\right|^{2}\left|\mu_{e_{m} g_{m}}\right|^{2} \Phi_{e_{n} g_{n}}\left(\omega_{p u}\right) \\
& \times \Phi_{e_{m} g_{m}}\left(\omega_{p r}\right)
\end{aligned}
$$

and

$$
\begin{aligned}
R_{4}^{(3)}\left(\omega_{p u}, \tau, \omega_{p r}\right)= & -\sum_{n m} p_{n}^{0} p_{m}(\tau)\left|\mu_{e_{n} g_{n}}\right|^{2}\left|\mu_{e_{m} g_{m}}\right|^{2} \Phi_{g_{n} e_{n}}\left(\omega_{p u}\right) \\
& \times \Phi_{e_{m} g_{m}}\left(\omega_{p r}\right) .
\end{aligned}
$$

Energy transfer transitions from quantum well $n$ to quantum well $m$ are incorporated into the $\mathrm{t}_{2}$-interval between fieldmatter interactions in the ESE response functions (see Fig. 14). The ESA signal components, which similarly account for energy transfer dynamics, are given by

$$
\begin{aligned}
R_{5}^{(3)}\left(\omega_{p u}, \tau, \omega_{p r}\right)= & \sum_{n m} p_{n}^{0} p_{m}(\tau)\left|\mu_{e_{n} g_{n}}\right|^{2}\left|\mu_{f_{m} e_{m}}\right|^{2} \Phi_{g_{n} e_{n}}\left(\omega_{p u}\right) \\
& \times \Phi_{f_{m} e_{m}}\left(\omega_{p r}\right)
\end{aligned}
$$

and

$$
\begin{aligned}
R_{6}^{(3)}\left(\omega_{p u}, \tau, \omega_{p r}\right)= & \sum_{n m} p_{n}^{0} p_{m}(\tau)\left|\mu_{e_{n} g_{n}}\right|^{2}\left|\mu_{f_{m} e_{m}}\right|^{2} \Phi_{e_{n} g_{n}}\left(\omega_{p u}\right) \\
& \times \Phi_{f_{m} e_{m}}\left(\omega_{p r}\right) .
\end{aligned}
$$

The signs of the response function represent the change in absorbance, $\triangle \mathrm{A}$. Resonances in the GSB and ESE correspond to $\Delta \mathrm{A}<0$, whereas the ESA response yields peaks with $\Delta \mathrm{A}>0$.

The transient absorption signals are obtained by summing over these six terms,

$$
S_{T A}\left(\omega_{p u}, \tau, \omega_{p r}\right) \equiv \sum_{j=1}^{6} R_{j}^{(3)}\left(\omega_{p u}, \tau, \omega_{p r}\right) .
$$

\section{APPENDIX B: ESTIMATION OF ENERGY TRANSFER RATE}

In this appendix, we compare the empirical rate constants to predictions based on the Förster model for energy transfer. ${ }^{20}$ To begin, transition dipole magnitudes of the quantum wells, $\mu$, can be derived from experimentally determined oscillator strengths, $f$, using ${ }^{55}$

$$
\mu^{2}=f\left(\frac{3 \hbar e^{2}}{2 m \omega}\right),
$$

where $\omega$ is the angular frequency associated with the excitonic transition. In order to estimate the energy transfer rate, we 
employ oscillator strengths of $f=0.3$ for the $\mathrm{n}=2$ and $\mathrm{n}=3$ quantum wells. ${ }^{11}$ The transition dipole corresponding to this oscillator strength is $15.6 \mathrm{D}$. The Coulomb coupling between transition dipoles for quantum wells 2 and 3 can be written $\operatorname{as}^{21}$

$$
J_{23}=\frac{5.04 \mathrm{~cm}^{-1} \mathrm{~nm}^{3}}{\mathrm{D}^{2}}\left[\frac{\vec{\mu}_{2} \cdot \vec{\mu}_{3}-3\left(\vec{\mu}_{2} \cdot \hat{n}_{23}\right)\left(\vec{\mu}_{3} \cdot \hat{n}_{23}\right)}{d_{23}^{3}}\right],
$$

where $\vec{\mu}_{k}$ is the transition dipole for quantum well $k, \hat{n}_{23}$ is a unit vector between the quantum wells, and $d_{23}$ is the distance between dipoles. The distance, $d_{23}$, must be greater than or equal to the 0.9 -nm thick organic layer. ${ }^{10,11}$ The constant prefactor in Eq. (B2) applies to transition dipoles with units of Debye and an inter-dipole distance in units of nanometers. The coupling is given by

$$
J_{23} \approx \frac{4.95 \mathrm{~cm}^{-1} \mathrm{~nm}^{3}}{\mathrm{D}^{2}}\left[\frac{\left|\vec{\mu}_{2}\right|\left|\vec{\mu}_{3}\right|}{d_{23}^{3}}\right],
$$

if we assume that the transition dipoles are coplanar and randomly oriented (see the supplementary material). We further assume that rotational diffusion of the transition dipoles is faster than the energy transfer time scale. ${ }^{56}$ Alternate constraints can be introduced for the time scale of dipole diffusion; however, these details will not significantly impact the present order-of-magnitude estimate for the energy transfer rate. We compute the distance, $d_{23}$, under the assumption that the transition dipoles are located in the centers of the lead-halide quantum wells using

$$
d_{23} \approx 0.9 \mathrm{~nm}+\frac{(2+3) 0.64 \mathrm{~nm}}{2},
$$

where the integers 2 and 3 in the numerator represent the indices of the quantum wells, and each I-Pb-I unit is assigned a 0.64-nm distance. ${ }^{5}$ With these parameters, the coupling strength, $J_{23}$, is equal to $75 \mathrm{~cm}^{-1}(9.3 \mathrm{meV})$.

The energy transfer rate can be computed with ${ }^{21}$

$$
K_{23}=\frac{J_{23}^{2}}{\hbar^{2}} \int_{-\infty}^{\infty} \tilde{\sigma}_{2}(\omega) \tilde{\sigma}_{3}(\omega) d \omega,
$$

where $\tilde{\sigma}_{2}(\omega)$ and $\tilde{\sigma}_{3}(\omega)$ are normalized optical spectra for quantum wells 2 and 3 , respectively. The line shape of the donor, $\tilde{\sigma}_{2}(\omega)$, generally incorporates nuclear relaxation via a Stokes shift. However, the Stokes shifts for the quantum wells are quite small because the peaks of the resonances differ by less than $100 \mathrm{~cm}^{-1}$ between the linear absorbance spectrum and the stimulated emission peaks in the transient absorption signals. We take the peaks of the resonances to be at 17544 and $16393 \mathrm{~cm}^{-1}$ (570 and $610 \mathrm{~nm}$ ) for quantum wells 2 and 3 , respectively. The resonances in the absorbance spectrum have FWHM linewidths of approximately $1000 \mathrm{~cm}^{-1}$. Evaluation of the integral in Eq. (B5) yields an overlap factor of 0.16 with these parameters. An energy transfer rate constant of $0.02 \mathrm{ps}^{-1}$ (time constant of $50 \mathrm{ps}$ ) is obtained by combining the coupling of $75 \mathrm{~cm}^{-1}(9.3 \mathrm{meV})$ with the overlap factor of 0.16 . Following the same approach, we obtain a rate constant of $0.005 \mathrm{ps}^{-1}$ (time constant of $200 \mathrm{ps}$ ) for quantum wells 3 and 4; here we have assumed an inter-dipole distance of
$3 \mathrm{~nm}$ and an oscillator strength of 0.3 for the $\mathrm{n}=4$ quantum well.

The estimated energy transfer time constants of 50 and $200 \mathrm{ps}$ are in good agreement with the averages of the measured values of 88 and $150 \mathrm{ps}$ (average of both systems); such agreement is noteworthy considering the idealized assumptions that must be made for this complex system. The dipole orientations, distances, and shapes of the transition densities can always be handled differently. Nonetheless, the present treatment firmly establishes the plausibility of energy transfer as the relaxation mechanism. This is important given the discrepancies in signal interpretation found in the recent literature. That is, photoinduced dynamics have been assigned to energy transfer in some systems, ${ }^{18}$ whereas others have suggested that electron transfer is the primary relaxation mechanism. ${ }^{15}$

${ }^{1}$ C. R. Kagan, D. B. Mitzi, and C. D. Dimitrakopoulos, Science 286, 945-947 (1999).

${ }^{2}$ M. M. Lee, J. Teuscher, T. Miyasaka, T. N. Murakami, and H. J. Snaith, Science 338, 643-647 (2012).

${ }^{3}$ M. A. Green, A. Ho-Baillie, and H. J. Snaith, Nat. Photonics 8, 506-514 (2014)

${ }^{4}$ J.-H. Yang, Q. Yuan, and B. I. Yakobson, J. Phys. Chem. C 120, 24682-24687 (2016).

${ }^{5}$ C. C. Stoumpos, D. H. Cao, D. J. Clark, J. Young, J. M. Rondinelli, J. I. Jang, J. T. Hupp, and M. G. Kanatzidis, Chem. Mater. 28, 2852-2867 (2016).

${ }^{6}$ J.-C. Blancon, H. Tsai, W. Nie, C. C. Stoumpos, L. Pedesseau, C. Katan, M. Kepenekian, C. M. M. Soe, K. Appavoo, M. Y. Sfeir, S. Tretiak, P. M. Ajayan, M. G. Kanatzidis, J. Even, J. J. Crochet, and A. D. Mohite, Science 355, 1288-1292 (2017).

${ }^{7}$ M. Grätzel, Acc. Chem. Res. 50, 487-491 (2017).

${ }^{8}$ T. Ishihara, J. Takahashi, and T. Goto, Solid State Commun. 69, 933-936 (1989).

${ }^{9}$ T. Ishihara, J. Takahashi, and T. Goto, Phys. Rev. B 42, 11099-11107 (1990).

${ }^{10}$ J. Calabrese, N. L. Jones, R. L. Harlow, N. Herron, D. L. Thorn, and Y. Wang, J. Am. Chem. Soc. 113, 2328-2330 (1991).

${ }^{11}$ X. Hong, T. Ishihara, and A. V. Nurmikko, Phys. Rev. B 45, 6961-6964 (1992).

${ }^{12}$ T. Umebayashi, K. Asai, T. Kondo, and A. Nakao, Phys. Rev. B 67, 155405 (2003).

${ }^{13}$ I. C. Smith, E. T. Hoke, D. Solis-Ibarra, M. D. McGehee, and H. I. Karunadasa, Angew. Chem., Int. Ed. 53, 11232-11235 (2014).

${ }^{14}$ O. Yaffe, A. Chernikov, Z. M. Norman, Y. Zhong, A. Velauthapillai, A. van der Zande, J. S. Owen, and T. F. Heinz, Phys. Rev. B 92, 045414 (2015).

${ }^{15}$ J. Liu, J. Leng, K. Wu, J. Zhang, and S. Jin, J. Am. Chem. Soc. 139, 1432-1435 (2017).

${ }^{16}$ R. MacColl and D. Guard-Friar, Phycobilisomes (CRC Press, Boca Raton, 1987).

${ }^{17}$ N. Wang, L. Cheng, R. Ge, S. Zhang, Y. Miao, W. Zou, C. Yi, Y. Sun, Y. Cao, R. Yang, Y. Wei, Q. Guo, Y. Ke, M. Yu, Y. Jin, Y. Liu, Q. Ding, D. Di, L. Yang, G. Xing, H. Tian, C. Jin, F. Gao, R. H. Friend, J. Wang, and W. Huang, Nat. Photonics 10, 699-704 (2016).

${ }^{18}$ M. Yuan, L. N. Quan, R. Comin, G. Walters, R. Sabatini, O. Voznyy, S. Hoogland, Y. Zhao, E. M. Beauregard, P. Kanjanaboos, Z. Lu, D. H. Kim, and E. H. Sargent, Nat. Nanotechnol. 11, 872-877 (2016).

${ }^{19}$ Q. Shang, Y. Wang, Y. Zhong, Y. Mi, L. Qin, Y. Zhao, X. Qui, X. Liu, and Q. Zhang, J. Phys. Chem. Lett. 8, 4431-4438 (2017).

${ }^{20}$ T. Förster, Ann. Phys. 437, 55-75 (1948).

${ }^{21}$ G. D. Scholes, Annu. Rev. Phys. Chem. 54, 57-87 (2003).

${ }^{22}$ S. Mukamel, Principles of Nonlinear Optical Spectroscopy (Oxford University Press, New York, 1995).

${ }^{23}$ S. Mukamel, Annu. Rev. Phys. Chem. 51, 691-729 (2000).

${ }^{24}$ D. M. Jonas, Annu. Rev. Phys. Chem. 54, 425-463 (2003).

${ }^{25}$ J. P. Ogilvie and K. J. Kubarych, Adv. At., Mol., Opt. Phys. 57, 249-321 (2009).

${ }^{26}$ D. B. Turner, K. E. Wilk, P. M. G. Curmi, and G. D. Scholes, J. Phys. Chem. Lett. 2, 1904-1911 (2011).

${ }^{27}$ B. Spokoyny and E. Harel, J. Phys. Chem. Lett. 5, 2808-2814 (2014).

${ }^{28}$ T. A. Gellen, L. A. Bizimana, W. P. Carbery, I. Breen, and D. B. Turner, J. Chem. Phys. 145, 064201 (2016). 
${ }^{29}$ M. K. Yetzbacher, N. Belabas, K. A. Kitney, and D. M. Jonas, J. Chem. Phys. 126, 044511 (2007).

${ }^{30}$ A. P. Spencer, H. Li, S. T. Cundiff, and D. M. Jonas, J. Phys. Chem. A 119, 3936-3960 (2015).

${ }^{31}$ P. Hamm, M. Lim, W. F. DeGrado, and R. M. Hochstrasser, Proc. Natl. Acad. Sci. U. S. A. 96, 2036-2041 (1999).

${ }^{32}$ J. F. Cahoon, K. R. Sawyer, J. P. Schlegel, and C. B. Harris, Science 319, 1820-1823 (2008).

${ }^{33}$ J. C. Wright, Annu. Rev. Phys. Chem. 62, 209-230 (2011).

${ }^{34}$ C. Consani, G. Auböck, F. van Mourik, and M. Chergui, Science 339, 1586-1589 (2013).

${ }^{35}$ M. E. F. Bouduban, A. Burgos-Caminal, R. Ossola, J. Teuscher, and J. E. Moser, Chem. Sci. 8, 4371-4380 (2017).

${ }^{36}$ H. Shi, R. Yan, S. Bertolazzi, J. Brivio, B. Gao, A. Kis, D. Jena, H. G. Xing, and L. Huang, ACS Nano 7, 1072-1080 (2013).

${ }^{37}$ Z. Nie, R. Long, L. Sun, C.-C. Huang, J. Zhang, Q. Xiong, D. W. Hewack, Z. Shen, O. V. Prezhdo, and Z.-H. Loh, ACS Nano 8, 10931-10940 (2014).

${ }^{38}$ K. J. Czech, B. J. Thompson, S. Kain, Q. Ding, M. J. Shearer, R. J. Hamers, S. Jin, and J. C. Wright, ACS Nano 9, 12146-12157 (2015).

${ }^{39}$ H. Fidder, J. Knoester, and D. A. Wiersma, J. Chem. Phys. 98, 6564-6566 (1993).

${ }^{40}$ M. Bednarz and J. Knoester, J. Phys. Chem. B 105, 12913-12923 (2001).

${ }^{41}$ M. B. Johnston and L. M. Herz, Acc. Chem. Res. 49, 146-154 (2016).

${ }^{42}$ G.-J. A. H. Wetzelaer, M. Scheepers, A. M. Sempere, C. Momblona, J. Ávila, and H. J. Bolink, Adv. Mater. 27, 1837-1841 (2015).
${ }^{43}$ P. Langevin, Ann. Chim. Phys. 28, 433-530 (1903).

${ }^{44}$ J. S. Manser and P. V. Kamat, Nat. Photonics 8, 737-743 (2014).

${ }^{45}$ Y. Yamada, T. Nakamura, M. Endo, A. Wakamiya, and Y. Kanemitsu, J. Am. Chem. Soc. 136, 11610-11613 (2014).

${ }^{46}$ Y. Yamada, T. Yamada, L. Q. Phuong, N. Maruyama, H. Nishimura, A. Wakamiya, Y. Murata, and Y. Kanemitsu, J. Am. Chem. Soc. 137, 10456-10459 (2015).

${ }^{47}$ A. R. Beattie and P. T. Landsberg, Proc. R. Soc. London, Ser. A 249, 16-29 (1959).

${ }^{48}$ A. Haug, J. Phys. C: Solid State Phys. 16, 4159-4172 (1983).

${ }^{49}$ J. M. Womick and A. M. Moran, J. Phys. Chem. B 113, 15747-15759 (2009).

${ }^{50}$ J. M. Womick and A. M. Moran, J. Phys. Chem. B 113, 15771-15782 (2009).

${ }^{51}$ M. H. Elkins, R. Pensack, A. H. Proppe, O. Voznyy, L. N. Quan, S. O. Kelley, E. H. Sargent, and G. D. Scholes, J. Phys. Chem. Lett. 8, 3895-3901 (2017).

${ }^{52} \mathrm{H}$. van Amerongen, L. Valkunas, and R. van Grondelle, Photosynthetic Excitons (World Scientific, Singapore, 2000).

${ }^{53}$ L. Valkunas, D. Abramavicius, and T. Mančal, Molecular Excitation Dynamics and Relaxation: Quantum Theory and Spectroscopy (WileyVCH, Weinheim, 2013).

${ }^{54}$ D. Abramavicius, B. Palmieri, D. V. Voronine, F. Sanda, and S. Mukamel, Chem. Rev. 109, 2350-2408 (2009).

${ }^{55}$ R. C. Hillborn, Am. J. Phys. 50, 982-986 (1982).

${ }^{56} \mathrm{~A}$. Tokmakoff, "Time-dependent quantum mechanics and spectroscopy," URL: http://tdqms.uchicago.edu, 2014. 